\title{
Revisiting substorm events with preonset aurora
}

\author{
Yukinaga Miyashita ${ }^{1}$ and Akimasa Ieda ${ }^{2}$ \\ ${ }^{1}$ Korea Astronomy and Space Science Institute, Daejeon, South Korea \\ ${ }^{2}$ Institute for Space-Earth Environmental Research, Nagoya University, Nagoya, Japan
}

Correspondence: Yukinaga Miyashita (miyasita@kasi.re.kr)

Received: 26 July 2017 - Revised: 28 September 2018 - Accepted: 8 October 2018 - Published: 19 October 2018

\begin{abstract}
Nishimura et al. (2010) proposed a new plasma intrusion or preonset aurora scenario of substorm triggering. In this scenario, a substorm is triggered by a fast earthward flow generated at the distant neutral line which corresponds to a preonset auroral streamer or arc in the ionosphere propagating from the auroral poleward boundary to the initial auroral brightening site, i.e., "preonset aurora". In the present paper, we revisited three substorm events reported as being triggered by such a mechanism related to preonset auroras, based on THEMIS ground-based all-sky imager data. Unlike previous studies, we examined the arrival timing of the preonset aurora relative to the three steps of auroral onset arc development (initial brightening, enhancement of the wavelike structure, and poleward expansion) to make the role of the preonset aurora in the auroral steps clearer. Our detailed timing analysis found that preonset auroral streamers reached the auroral onset arc but away from the initial brightening site after initial brightening for two events, while no preonset aurora reaching the initial brightening site could be identified for the other event. This result suggests that the processes associated with auroral streamers are unlikely to affect at least initial brightening, even if we consider not only the presence and arrival timing and location of the auroral streamers but also the scale of the corresponding flow and flow vortices. We list a series of open questions for testing the preonset aurora scenario further in future studies.
\end{abstract}

Keywords. Magnetospheric physics (storms and substorms; auroral phenomena; magnetotail)

\section{Introduction}

The substorm triggering mechanism is still one of the most controversial issues in magnetospheric research. Substorm models proposed so far include the near-Earth neutral line (NENL) model (e.g., Baker et al., 1996), the current disruption (CD) model (e.g., Lui, 1996), and the catapult (slingshot) current-sheet relaxation model (Machida et al., 2009, 2014). These models are different in the physical process and location of initial action associated with substorm onset and causal links between the substorm processes. That is, in the NENL model, magnetic reconnection generates a tailward moving plasmoid and a fast earthward flow at $X \sim-20$ $R_{\mathrm{E}}$ a few minutes before substorm onset. This earthward flow leads to current disruption and dipolarization at $X \sim-10 R_{\mathrm{E}}$ and auroral breakup. In the CD model, current disruption and dipolarization first occur at $X \sim-10 R_{\mathrm{E}}$, leading to auroral breakup and near-Earth reconnection. In the catapult currentsheet relaxation model, an imbalance between the $\boldsymbol{J} \times \boldsymbol{B}$ and pressure gradient forces generates a fast earthward flow in the near-Earth magnetotail before onset. This effect leads to near-Earth reconnection and current disruption just tailward and earthward of the imbalance region, respectively.

Furthermore, a series of papers by Nishimura et al. (2010, 2011, 2013c) and Lyons et al. (2010) proposed a new substorm model in which a new plasma intrusion linked to preonset aurora plays an important role in substorm triggering. That is, a fast earthward flow generated at the distant neutral line typically located at $X \sim-130 R_{\mathrm{E}}$ (see Machida et al., 2000), rather than at the near-Earth neutral line typically located at $X \sim-20$ to $-30 R_{\mathrm{E}}$ (see Nagai et al., 1998; Machida et al., 1999; Miyashita et al., 2000, 2009; Imber et al., 2011), reaches the near-Earth region at $X \sim-10 R_{\mathrm{E}}$ and triggers a substorm. This fast flow in the magnetotail corresponds to a preonset north-south auroral streamer or east- 
west auroral arc in the ionosphere propagating equatorward from the auroral poleward boundary, which they call preonset aurora. After the preonset aurora reaches the equatorward portion of the auroral oval, the onset (initial brightening) occurs at this location for about half of substorm events. For most of the rest of substorm events, the preonset aurora changes direction to move azimuthally, and initial brightening occurs when the preonset aurora reaches the initial brightening site. According to Nishimura et al. (2010), such preonset auroras related to initial brightening are observed frequently, i.e., for $\sim 90 \%$ of substorm events in total.

Many studies (e.g., Henderson, 2009; Rae et al., 2009; Mende et al., 2011; Murphy et al., 2014b; Kepko, 2014), however, reported substorm events without any auroral streamers and arcs moving to the auroral onset arc before initial auroral brightening and even poleward expansion. This indicates that the processes associated with a preonset aurora are not necessary conditions for substorm triggering. Thus the preonset aurora scenario proposed by Nishimura et al. (2010) is controversial, and more detailed reexaminations of their events are required to prove or disprove it.

In the present study, using the same auroral data as Nishimura et al. (2010, 2011), i.e., data from white-light ground-based observatory (GBO) all-sky imagers (ASIs) (Donovan et al., 2006; Mende et al., 2008) of the Time History of Events and Macroscale Interactions during Substorms (THEMIS) mission (Angelopoulos, 2008) at $3 \mathrm{~s}$ resolution, we revisited three substorm events that Nishimura et al. $(2010,2011)$ identified as isolated onset events triggered by the processes associated with preonset auroras. These events were discussed at a focus group, "Testing Proposed Links between Mesoscale Auroral and Polar Cap Dynamics and Substorms", of the summer and mini workshops of the Geospace Environment Modeling (GEM) program held in Snowmass, Colorado, and San Francisco, California, respectively, in 2015. Considering the present results, we list a series of open questions for testing the preonset aurora scenario further in future studies.

Here we performed more detailed analysis than Nishimura et al. $(2010,2011)$. The main differences between the respective approaches are as follows. The first is consideration of stepwise auroral onset arc development. Nishimura et al. $(2010,2011)$ considered only either initial brightening or poleward expansion as substorm onset. On the other hand, we attempted to distinguish the three steps of initial brightening, enhancement of the wave-like structure, and poleward expansion, as described below. This will make our discussion about the arrival timing of the preonset aurora relative to the three auroral steps and the role of the preonset aurora in the auroral steps clearer. The second difference is the timing determination method. Nishimura et al. $(2010,2011)$ determined the timings of the substorm onset and the streamer arrival visually from substantial intensification. On the other hand, we determined the timings of the auroral onset arc development and the streamer arrival by a more quantitative method, pay- ing attention to initial faint auroras as well, as described in the next section. The third difference is consideration of the spatial relationship between the auroral onset arc, the auroral streamer, and the associated flow channel and flow vortices, as described below. Nishimura et al. $(2010,2011)$ considered the flow channel, but we considered the flow vortices as well.

Before describing the three events, we summarize stepwise development of the auroral onset arc whose timings we determined for the present examination. The auroral arc develops in four steps in association with substorm onset (Mende et al., 2009): (1) preonset fading, (2) initial brightening, (3) enhancement of the wave-like structure, and (4) poleward expansion. (1) Although in not all substorms, preonset auroral fading or dimming may be seen a few minutes before initial auroral brightening or breakup (Pellinen and Heikkila, 1978; Kauristie et al., 1997; Murphy et al., 2012, 2013). This phenomenon does not necessarily extend to the entire arc, but is localized, which is consistent with localized decrease in upward field-aligned currents (Murphy et al., 2012, 2013; Coxon et al., 2017). We should note that $630.0 \mathrm{~nm}$ emission related to low-energy $(<\sim 1 \mathrm{keV})$ electrons intensified during fading of $557.7 \mathrm{~nm}$ emission primarily related to high-energy $(>\sim 1 \mathrm{keV})$ electrons (Deehr and Lummerzheim, 2001). Deehr and Lummerzheim (2001) inferred that this difference between the emissions is a signature of Alfvén waves, which is possibly supported by the result of Chaston et al. (2002) that electrons accelerated by Alfvén waves are in a lower energy range. (2) An auroral arc begins to intensify or newly appears at initial brightening. The brightening segment is localized to a longitudinal width of $\sim 30-60 \mathrm{~km}$ at the very early stage (Sakaguchi et al., 2009a; see also Nishimura et al., 2016). It then extends westward and eastward, and a wave-like or bead-like structure grows gradually (e.g., Davis, 1962; Elphinstone et al., 1995; Friedrich et al., 2001; Donovan et al., 2007; Liang et al., 2008; Sakaguchi et al., 2009a, b; Henderson, 2009; Rae et al., 2009, 2010; Kepko et al., 2009; Motoba et al., 2012, 2015; Chang et al., 2012; Murphy et al., 2014b; Chang and Cheng, 2015; Kalmoni et al., 2015, 2017; Motoba and Hirahara, 2016; Nishimura et al., 2016). Akasofu (1964) described initial brightening as being sudden, but this intensification seems to be gradual and can last for several minutes (Lyons et al., 2002; Liang et al., 2008; Mende et al., 2009; Sakaguchi et al., 2009b). This discrepancy of sudden or gradual intensification possibly comes from the time resolution and sensitivity of cameras used. (3) Then the luminosity of the arc is enhanced exponentially at some time and the wavelike structure becomes clearer and grows further. Mende et al. (2009) described this stage as appearance of a new rayed arc, but we call it enhancement of the wave-like structure here. (4) Finally, poleward expansion or auroral breakup begins.

In spite of stepwise auroral development, most previous studies marked only one or at most two timings of the latter three auroral timings and chose one as the substorm onset 
time. That is, different studies adopted different definitions of the substorm onset. Some studies adopted initial brightening as the substorm onset, while others adopted enhancement of the wave-like structure or poleward expansion. Nishimura et al. $(2010,2011)$ intended to mark only initial brightening as the substorm onset, while Nishimura et al. (2016) marked initial brightening and poleward expansion. Thus, not to lead to confusion, we will avoid using the term "onset" without caution when we describe the timings of auroral onset arc development below.

Each step of auroral development probably corresponds to magnetotail substorm signatures. That is, Miyashita et al. (2015) suggested that initial brightening possibly corresponds to near-Earth magnetic reconnection, enhancement of the wave-like structure corresponds to growth of the ballooning instability in the near-Earth magnetotail, and poleward expansion corresponds to near-Earth dipolarization. This indicates that determining and distinguishing all of the three steps in particular are very important for making our discussions about the timing issue and the magnetotail development clear. In the case of the present study, examining the arrival timing of the preonset aurora relative to the three auroral steps (initial brightening, enhancement of the wave-like structure, and poleward expansion) is expected to make the role of the preonset aurora in the auroral steps clearer.

In addition to the timing of each auroral step, we should pay attention to the spatial relationship between the auroral streamer and the associated flow channel and flow vortices. Nishimura et al. $(2010,2011)$ considered the flow channel, but they do not seem to have considered the flow vortices. As shown in the schematic of Fig. 1, the auroral streamer corresponds, not to the central part of the flow channel, but to the western edge of the flow channel, i.e., the western flow vortex and upward field-aligned current (Kauristie et al., 2000; Nakamura et al., 2001). A typical scale of the flow vortex is up to $\sim 1 \mathrm{~h}$ in magnetic local time (MLT) in the ionosphere (Amm et al., 1999; Kauristie et al., 2000; Nakamura et al., 2001). Hence, considering not only the auroral streamer but also the central part of the flow and the flow vortices, the separation between the auroral streamer and the initial brightening site should be within the sum of the scale of the eastern flow vortex and the flow channel width if initial brightening occurs east of the auroral streamer. On the other hand, it should be within the scale of the western flow vortex if initial brightening occurs west of the auroral streamer. If the separation is more than these scales, the processes associated with the streamer would not affect initial brightening. Furthermore, considering the angle between the auroral streamer and the onset arc, if the auroral streamer moves purely equatorward, the auroral streamer, the central part of the flow, and the flow vortices may arrive at the onset arc simultaneously (Fig. 1a). If the auroral streamer moves equatorward and westward, the central part of the flow and the eastern flow vortex may contact the onset arc earlier than the auroral streamer (the western flow vortex) (Fig. 1b). If the au- roral streamer moves equatorward and eastward, the auroral streamer may contact the onset arc earlier than the central part of the flow and the eastern flow vortex (Fig. 1c).

\section{Timing determination method}

To determine the timings of the auroral development associated with substorm onsets, different previous studies adopted different methods. In studies examining not only two-dimensional auroral images but also luminosity curves from THEMIS ASI, for example, Mende et al. (2009) determined the three timings of initial brightening, enhancement of the wave-like structure, and poleward expansion from slope increase (breakpoint) in the total integrated luminosity curve, although they seem to have identified visually. Angelopoulos et al. (2008), Gabrielse et al. (2009), and Liu et al. (2011) used a similar method to determine only auroral intensification time from the breakpoint of the integrated auroral luminosity over the region of interest. This intensification occurred before poleward expansion, so it most likely corresponds to initial brightening or enhancement of the wavelike structure. Murphy et al. (2014a) also used the total integrated luminosity, but attempted automatic determination of the auroral breakup interval, which corresponds to what Angelopoulos et al. (2008) determined. Nishimura et al. (2016) determined initial brightening and poleward expansion. They defined the former by the initial rise of the maximum luminosity along the onset arc, which is simultaneous with the beginning of growth of the onset arc's wave-like structure.

Kalmoni et al. $(2015,2017)$ determined two auroral timings by different quantitative methods considering development of the wave-like (bead-like) auroral structure. Kalmoni et al. (2015) first identified clear appearance of the wavelike structure visually and then determined the beginning of growth of the wave-like structure with linear fitting in log space for individual wave numbers. Kalmoni et al. (2017) determined the beginning of growth of the wave-like structure on the basis of the appearance of the wave-like structure and also determined that of exponential growth of the total auroral luminosity with linear fitting in log space. In spite of the different methods, their total luminosity curve and north-south and east-west keograms indicate that the first and second timings most likely correspond to initial brightening and enhancement of the wave-like structure, respectively, and poleward expansion occurred at a later time.

Thus quantitative timing determination in the previous studies is based on mainly the breakpoint (trend increase) of the luminosity curve and growth of the wave-like structure. In the present study, we adopted the former for the following reasons, developing the previous method as described below. Our method can determine all the timings of the three auroral steps of initial brightening, enhancement of the wave-like structure, and poleward expansion that Mende et al. (2009) proposed, although it is simple. As Nishimura et al. (2016) 
(a)

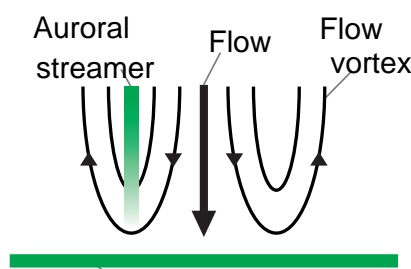

Auroral onset arc (b)

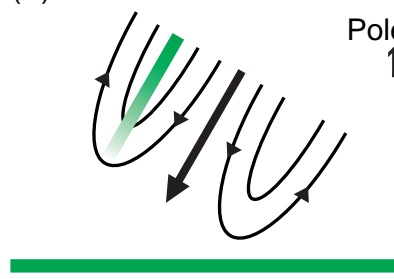

(c)

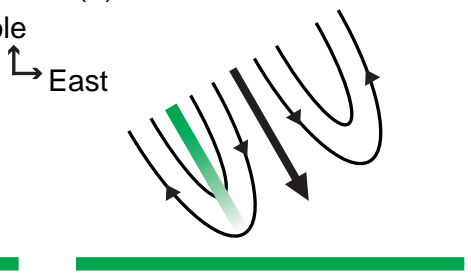

Figure 1. Schematics showing the spatial relationship between the auroral onset arc and the concurrent flow channel and flow vortices in the cases of (a) a purely equatorward moving streamer, (b) an equatorward and westward moving streamer, and (c) an equatorward and eastward moving streamer.

and Kalmoni et al. (2017) showed, the auroral luminosity begins to increase at the same time as growth of the onset arc's wave-like structure. Although growth of the wave-like structure is an important feature of the auroral onset arc, determining the trend increases in the luminosity is adequate for our purpose of timing discussion.

Our method of timing determination is as follows (the results of each event are described in detail in the next section). We first determined the timing and location of the three auroral steps visually from two-dimensional ASI images (as Fig. 2 and Supplement Movie S1) by changing the blackwhite scale to pay attention to initial faint auroras as well and looking at the series of the images back and forth. To see the variations in the luminosity of the auroral arc and determine the timings of auroral onset arc development quantitatively, we then examined the variations of average counts for areas of $0.5^{\circ}$ longitudinal width including the auroral onset arc (the magenta boxes in Fig. 2), as shown in Fig. 3. To determine the trend increase in the luminosity, we applied a segmented (piecewise) linear fitting with one breakpoint (cf. Tomé and Miranda, 2004, 2005) to the time-series count data for each area at and near the location of each step around its beginning. Here we used the Levenberg-Marquardt algorithm for the fitting (Moré, 1978; Markwardt, 2009). Whether two lines with one breakpoint or a single line are better fitting was judged from the values of $\chi^{2}$. We took fitting intervals manually. Unless they are too short and include the previous and next large changes, different intervals did not make large differences in the fitting results. In Fig. 3, the thick line for each area indicates a result of the fitting. The short vertical bar indicates the breakpoint of the line, while it is not drawn if a single straight line is better in fitting. The horizontal bar at the breakpoint indicates the error of the time of the breakpoint. For each auroral step, the earliest breakpoint where the slope increased is regarded as the beginning of the step. If two auroral steps are close in time to each other, as in Fig. 3b, the two fitting intervals are allowed to overlap with each other to make the fitting intervals not too short. Furthermore, we tried this method on both linear and logarithmic scales. We found that the method worked and the results were nearly the same between the linear and logarithmic scales for initial brightening and enhancement of the wave-like structure, but the method seems to have worked only for the logarithmic scale for poleward expansion (not shown). Hence we use the logarithmic scale for timing determination.

In addition, we adopted the same method as auroral onset arc development to auroral streamers. After determining the timing and location of auroral streamers visually from two-dimensional ASI images (as Fig. 2 and Supplement Movie S1) in a similar way to auroral onset arc development, we applied the segmented linear fitting with one breakpoint to each area of $0.05^{\circ}$ latitudinal width in the magenta box indicated in two-dimensional images including the streamer's path from the poleward arc or a few degrees poleward of the auroral onset arc to the poleward part of the auroral onset arc (Fig. 4). The count should increase when a streamer enters an area, and this increase should propagate equatorward until the streamer arrives just poleward of or at the auroral onset arc.

\section{Observations of auroral streamers}

We show only the results of ASI data analysis for the three events in this section, since they are not necessarily good conjunction events in terms of the locations of the THEMIS spacecraft in the magnetotail. We first describe the three timings of auroral onset arc development and then describe equatorward moving auroral streamers observed by ASIs.

\subsection{February 2008 event}

According to Nishimura et al. (2010, 2011), this event was an isolated substorm with poleward expansion. Figure 2 presents selected ASI images from Gillam (GILL), Canada $\left(66.00^{\circ}\right.$ geomagnetic latitude, $333.19^{\circ}$ geomagnetic longitude) (see also Supplement Movie S1). Figure 3 presents the variations of auroral counts along the auroral onset arc in the magenta boxes in the 05:22:39 and 05:28:00 UT panels of Fig. 2. Initial brightening and later development were observed in the field of view of this camera. Before initial brightening, the luminosity was increased gradually (Fig. 3a); such gradual intensification of the growth phase 


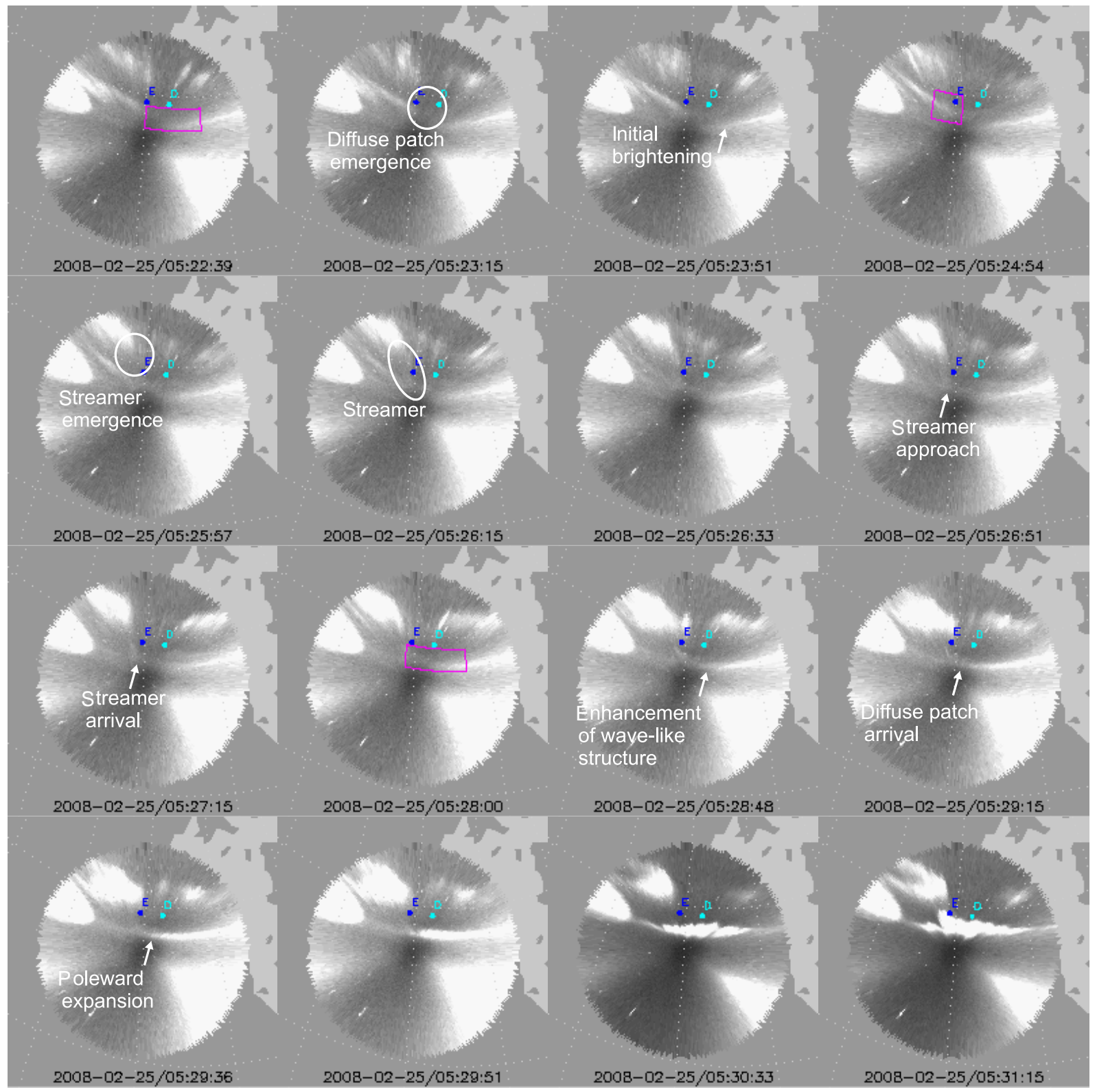

Figure 2. Selected auroral images obtained from the THEMIS GBO all-sky imager at GILL between 05:22 and 05:32 UT on 25 February 2008, along with footprints of THEMIS D (light blue) and E (blue) calculated using the T96 magnetic field model (Tsyganenko, 1995). White arrows and circles indicate approximate locations of auroral phenomena. The maximum count values of the black-white scale are 1800 for $05: 22: 39$ to $05: 29: 51$ UT and 3000 for the later times.

arc was also reported by Lessard et al. (2007). Preonset auroral fading was not seen just before initial brightening in the present event as well as in the other two events shown below. Although there was a bright arc extending from the eastern edge of the field of view toward the central part of the field of view, initial brightening did not occur on this arc, but instead occurred near the central part of the field of view at $\sim 68^{\circ}$ magnetic latitude and $\sim 23 \mathrm{~h}$ MLT at 05:23:50 UT. Figure 3a shows that the breakpoint due to slight increase in the trend appeared first at a localized segment (on yellow and orange lines) at this time and then appeared at the westward and eastward segments (on neighbors) successively. That is, the onset arc gradually grew brighter, although very faint, and it extended mainly westward and slightly eastward. This localization and gradual progress of initial brightening is consistent with Mende et al. (2009) and Sakaguchi et al. (2009a, b), as mentioned above. Figure $3 \mathrm{a}$ also shows that the luminosity oscillated after initial brightening by increasing the trend, due to gradual growth of the wave-like structure of the auroral onset arc. At 05:28:47 UT, $\sim 5$ min after initial 

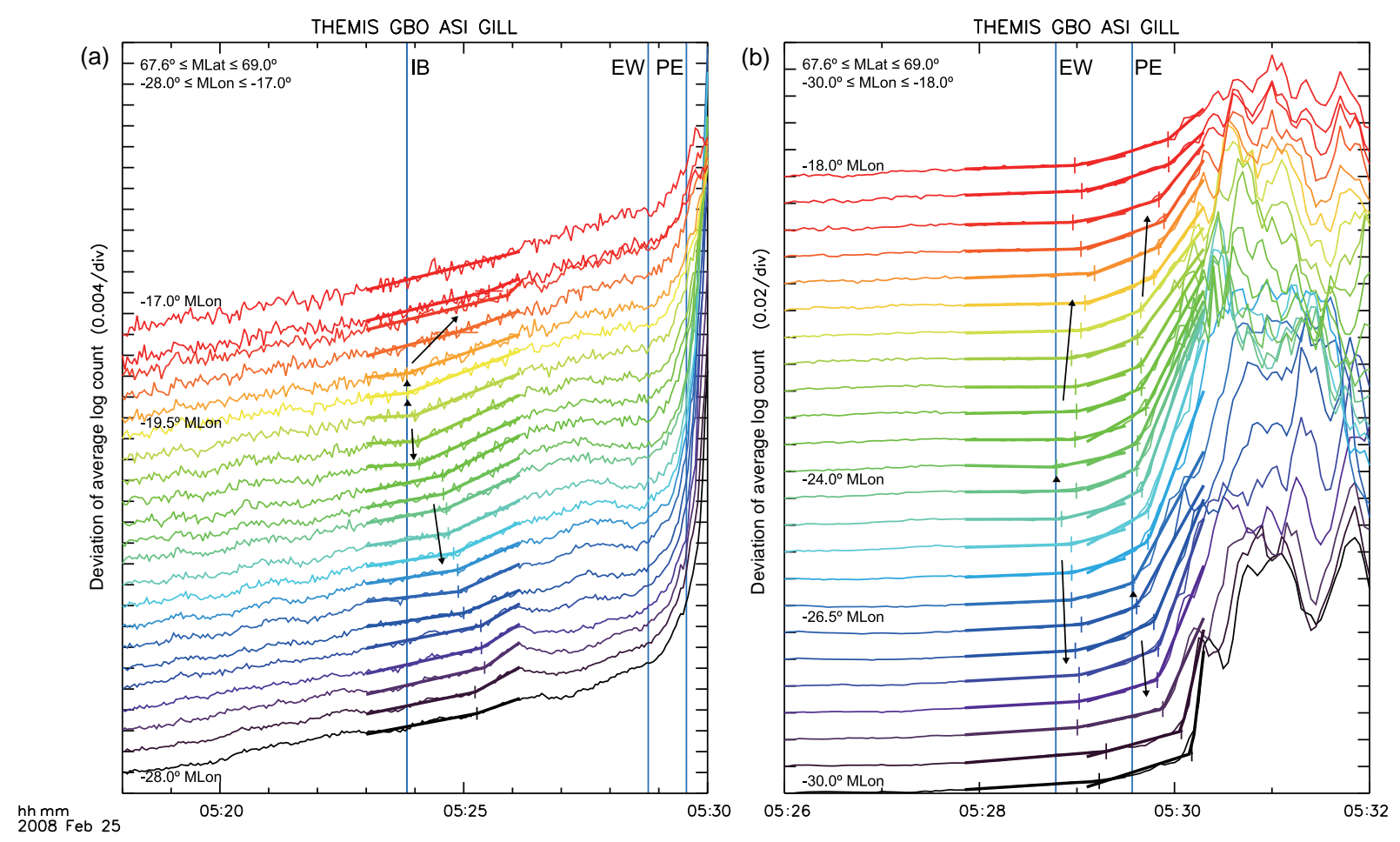

Figure 3. Variations of the averages of the logarithm of count for areas of $0.5^{\circ}$ longitudinal width in the region indicated in the top of this figure, or the magenta boxes in the (a) 05:22:39 and (b) 05:28:00 UT panels of Fig. 2, including the central and eastern parts of the auroral onset arc obtained from the THEMIS GBO all-sky imager at GILL on 25 February 2008. The data from the east to the west are plotted from the top to the bottom. The vertical lines with labels of IB, EW, and PE indicate the times of initial auroral brightening, enhancement of the wave-like structure, and poleward expansion, respectively. The thick line for each area at and near the site of each auroral step indicates a result of segmented (piecewise) linear fitting with one breakpoint. The short vertical bar indicates the breakpoint of the line, while it is not drawn if a single straight line is better in fitting. The horizontal bar at the breakpoint indicates the error of the time of the breakpoint. For each auroral step, the earliest breakpoint where the slope increased is regarded as the beginning of the step.

brightening, the onset arc as well as the wave-like or beadlike structure were further enhanced to the west of the initial brightening site. The breakpoint due to larger increase appeared first on a green line and then on neighbors in Fig. 3b. At 05:29:34 UT, another $\sim 50$ s later, poleward expansion began nearly at the same place as enhancement of the wave-like structure. The breakpoint due to explosive increase appeared first on a blue line and then on neighbors in Fig. 3b. The times of auroral onset arc development, along with those of the preonset aurora shown below, are summarized in Table 1 .

A few previous papers studied this event. Nishimura et al. (2010, 2011) determined only one timing of 05:29 UT, $\sim 5$ min later than our initial brightening time, for auroral onset arc development. Their timing is based on visual inspection of substantial intensification of the auroral onset arc, so it possibly corresponds to what we identified as enhancement of the wave-like structure. Kepko et al. (2009) also analyzed this event using multiple emission data. They determined the times of brightening of equatorward boundary, formation of auroral ray, auroral beading, and poleward expansion as 05:29:11, 05:29:43, 05:30:07, and 05:30:31 UT, respectively. Their first step possibly corresponds to initial brightening, while the second or third step corresponds to enhancement of the wave-like structure. Their timings are all later than our determination, possibly because they determined the timings from sufficiently intensified signatures, as also pointed out by Lui (2011). That is, Lui (2011), who revisited this event using the same data as Kepko et al. (2009), paid attention to faint aurora and pointed out that the luminosity was increasing from 05:26:50 to 05:29:14 UT. Although it is not clear whether he examined the data for earlier times, he determined the times of initial brightening and poleward expansion as between 05:26:37 and 05:27:01, and 05:29:35 UT, respectively. The latter agrees with our determination. Our interpretations of these times are summarized in Table 1.

The white-light images in Fig. 2 and Movie S1 show that the auroral streamer of this event was very faint. It seems to have appeared in the arc in the northern part of the field of view at $\sim 05: 25 \mathrm{UT}$ and then propagated in the equatorward and slightly eastward directions. The auroral count variations along the path of this streamer in Fig. 4 show that this streamer arrived just poleward of the onset arc at 05:26:50 UT, or $3 \mathrm{~min}$ after initial brightening and $\sim 2 \mathrm{~min}$ before enhancement of the wave-like structure (green lines 

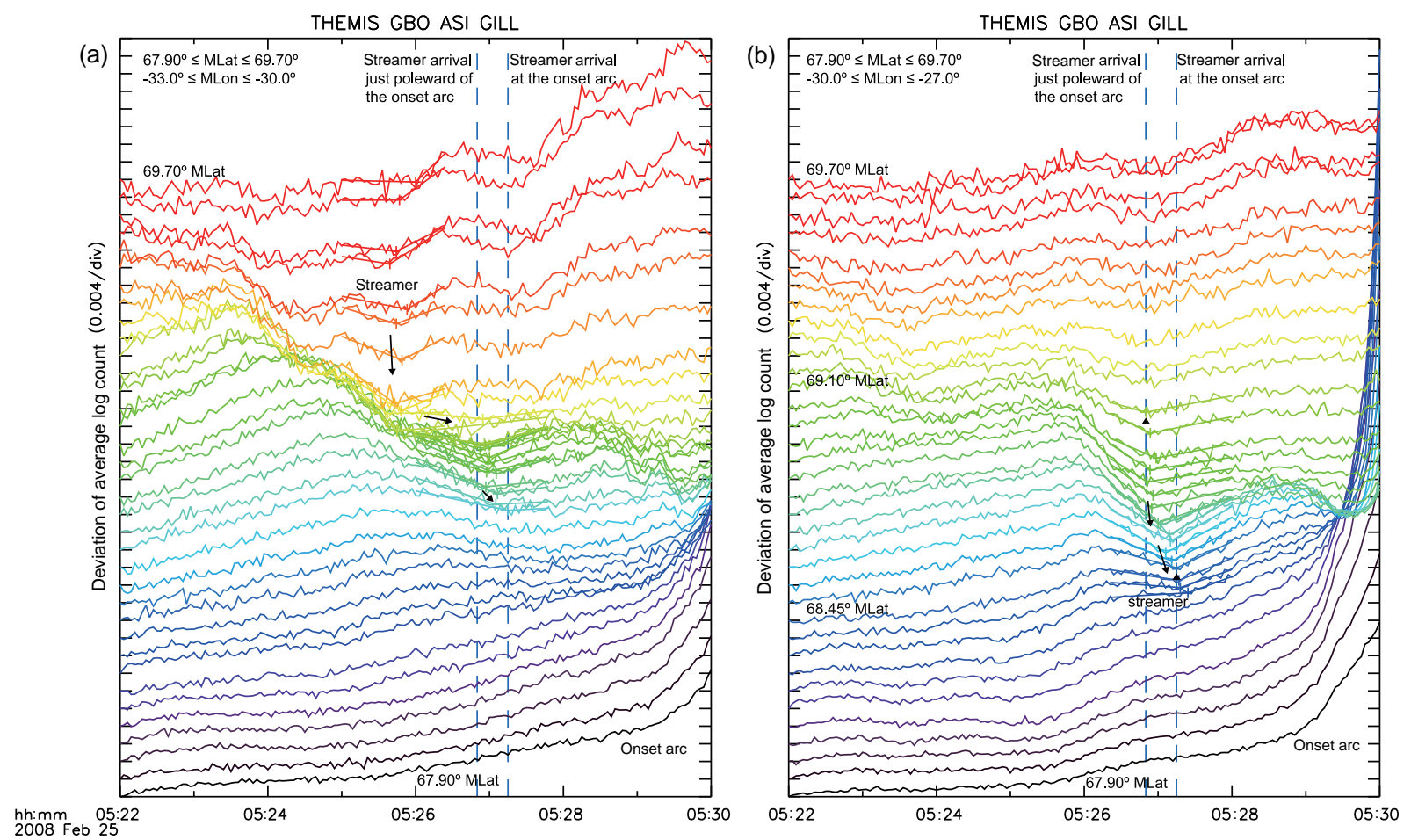

Figure 4. Variations of the averages of the logarithm of count for areas of $0.05^{\circ}$ latitudinal width in the region indicated in the top of this figure, or the (a) western and (b) eastern halves of the magenta box in the 05:24:54 UT panel of Fig. 2, including the path of the southeastward moving auroral streamer obtained from the THEMIS GBO all-sky imager at GILL on 25 February 2008. The data from the north to the south are plotted from the top to the bottom. The left and right vertical dashed lines indicate the times of the arrival of the streamer just poleward of and at the auroral onset arc, respectively. The thick line for each area where the streamer reached indicates a result of segmented (piecewise) linear fitting with one breakpoint, which indicates the time of the arrival of the streamer at this area. The short vertical bar indicates the breakpoint of the line, while it is not drawn if a single straight line is better in fitting. The horizontal bar at the breakpoint indicates the error of the time of the breakpoint.

in Fig. 4b). It then slowed down and arrived at the onset arc at 05:27:15 UT, or $\sim 3.5 \mathrm{~min}$ after initial brightening and $\sim 1.5 \mathrm{~min}$ before enhancement of the wave-like structure (blue lines in Fig. $4 \mathrm{~b}$ ). The arrival point was $\sim 0.3 \mathrm{~h}$ in MLT to the west of the initial brightening site as well as enhancement of the wave-like structure and poleward expansion. Note that after the auroral streamer arrived at the onset arc, it did not move along the onset arc. We also could not identify another preonset aurora moving along the onset arc from the outside of the field of view toward the initial brightening site.

According to Kepko et al. (2009), a diffuse auroral patch or streamer moving equatorward, which was seen clearly in their auroral images of $630.0 \mathrm{~nm}$ emission, emerged near the middle of the field of view at 05:23:15 UT and arrived at the onset arc at 05:29:14 UT, $\sim 30 \mathrm{~s}$ after enhancement of the wave-like structure and $20 \mathrm{~s}$ before poleward expansion. The western edge of this diffuse patch corresponds to a narrow discrete form seen in 557.7 and $427.8 \mathrm{~nm}$ emissions or the streamer mentioned above. As also pointed out by Lui (2011), Kepko et al. (2009) did not discuss that this streamer already connected with the onset arc at $\sim 05: 27$ UT. Further- more, Nishimura et al. (2013a) also examined this event. Although they did not describe the specific timings, they concluded that a polar cap patch was generated on the dayside, propagating across the polar cap and the nightside auroral poleward boundary, and then became the auroral streamer and/or diffuse patch that we and Kepko et al. (2009) discussed.

\subsection{February 2008 event}

Nishimura et al. (2011) showed this isolated substorm with poleward expansion in detail. The Dst and AE indices indicate that this substorm occurred during active time, or between two weak storms under enhanced convection. Figure 5 shows selected ASI images from McGrath (MCGR), Alaska (61.74 ${ }^{\circ}$ magnetic latitude, $260.25^{\circ}$ magnetic longitude) (see also Supplement Movie S2). The average count variations along the auroral onset arc (the magenta box in the 10:57:24 UT panel of Fig. 5) are shown in Fig. 6. Before initial brightening, the luminosity was increased gradually (Fig. 6a), similarly to the events of Lessard et al. (2007). Initial brightening occurred in the east of the field of view 
Table 1. UT of auroral onset arc development and preonset aurora that the present study determined by fitting, our interpretations of the times that previous studies determined, and the arrival location of the preonset aurora relative to the initial brightening or poleward expansion site ( $\triangle \mathrm{MLT}$ ) for the 25 February 2008 substorm event.

\begin{tabular}{|c|c|c|}
\hline 25 Feb 2008 & The present study & Previous studies \\
\hline \multicolumn{3}{|l|}{ Auroral onset arc development } \\
\hline \multirow[t]{2}{*}{ Initial brightening } & $05: 23: 50$ & 05:26:37-05:27:01 (Lui, 2011) \\
\hline & & 05:29:11 (Kepko et al., 2009) \\
\hline \multirow[t]{2}{*}{ Enhancement of the wave-like structure } & $05: 28: 47$ & 05:29 (Nishimura et al., 2010, 2011) \\
\hline & & 05:29:43 or 05:30:07 (Kepko et al., 2009) \\
\hline \multirow[t]{2}{*}{ Poleward expansion } & $05: 29: 34$ & 05:29:35 (Lui, 2011) \\
\hline & & 05:30:31 (Kepko et al., 2009) \\
\hline \multicolumn{3}{|l|}{ Preonset aurora } \\
\hline Emergence & $\sim 05: 25$ & diffuse patch, 05:23:15 (Kepko et al., 2009) \\
\hline \multirow[t]{2}{*}{ Arrival just poleward of/at the onset arc } & $05: 26: 50 / 05: 27: 15$ & $\begin{array}{l}\text { arrived (Nishimura et al., 2010, 2011, 2013a) } \\
\sim 05: 27 \text { (Lui, 2011) }\end{array}$ \\
\hline & & diffuse patch, 05:29:14 (Kepko et al., 2009) \\
\hline$\triangle \mathrm{MLT}, \mathrm{h}$ & $\sim 0.3$, west & \\
\hline
\end{tabular}

at $\sim 63^{\circ}$ magnetic latitude and $\sim 0 \mathrm{~h}$ MLT at 10:59:22 UT. Figure 6 a shows that the breakpoint due to slight increase in the trend appeared first at a localized segment (on a green line) at this time and then appeared at the westward segments (on neighbors) successively. That is, the onset arc gradually grew brighter and extended mainly westward. Here the luminosity enhancements west of the initial brightening site (in the west of the magenta box in Fig. 5) between $\sim 10: 58$ and 11:01 UT (blue to light violet lines in Fig. 6a) and between $\sim$ 11:00 and 11:02 UT (dark violet to black lines in Fig. 6a) were due to auroral streamers coming from the poleward region. Figure 6a also shows that the luminosity oscillated after initial brightening by increasing the trend, due to gradual growth of the wave-like structure of the auroral onset arc. At 11:07:19 UT, $\sim 8$ min after initial brightening, the onset arc as well as the wave-like structure were further enhanced in the middle of the field of view. The breakpoint due to larger increase appeared in this part of the onset arc (on a blue line and then on neighbors) in Fig. 6a and b. At 11:10:19UT, another $3 \mathrm{~min}$ later, poleward expansion began to the east of enhancement of the wave-like structure. The breakpoint due to explosive increase appeared first on a green line and then on neighbors in Fig. 6b. The times of auroral onset arc development are summarized in Table 2.

Nishimura et al. $(2010,2011)$ marked 11:10 UT from substantial intensification of the auroral onset arc. This timing is $\sim 10.5$ and 3 min after our times of initial brightening and enhancement of the wave-like structure, respectively, or rather, near our poleward expansion time. Hence we surmise that the time marked by Nishimura et al. $(2010,2011)$ was that of poleward expansion. Here note that another sudden auroral enhancement did not occur between initial brightening and enhancement of the wave-like structure.

A clear equatorward moving auroral streamer appeared between the poleward and equatorward $\operatorname{arcs}\left(\sim 1^{\circ}\right.$ poleward of the equatorward arc) in the middle of the field of view at 10:57:30 UT, although Nishimura et al. (2011) did not mention it. While this aurora was growing bright, it extended westward, or another aurora appeared on the westward side. These auroras moved equatorward, and the first auroral streamer arrived at the equatorward arc at $\sim 10: 58 \mathrm{UT}, \sim$ $1 \mathrm{~min}$ before initial brightening. The arrival point was $\sim 0.6 \mathrm{~h}$ in MLT west of the initial brightening site, but it may be within the expected flow vortex scale. Since this aurora appears to have been generated in the auroral oval, we may conclude that it was not related to Nishimura et al.'s (2010) scenario in which a preonset aurora should be generated at the auroral poleward boundary corresponding to the distant neutral line. We would like to report on this streamer in detail elsewhere.

After that, another very clear auroral streamer that Nishimura et al. (2011) regarded as being related to the processes leading to initial brightening appeared in the poleward part of the auroral oval after initial brightening at $\sim$ 11:07 UT and then extended toward the onset arc. The auroral count variations along the path of this streamer in Fig. 7 show that this streamer arrived just poleward of the onset arc near the site of enhancement of the wave-like structure at 11:07:50 UT, or $\sim 8.5 \mathrm{~min}$ after initial brightening and $\sim 30 \mathrm{~s}$ after enhancement of the wave-like structure (a light blue line). It then slowed down and arrived at the onset arc at 11:09:09 UT, or $\sim 2$ min after enhancement of the wavelike structure and $\sim 1$ min before poleward expansion (blue lines). The arrival point was $\sim 0.5 \mathrm{~h}$ or less in MLT to the west of the initial brightening site as well as enhancement of the wave-like structure and poleward expansion. Note that the streamer did not move eastward or toward the poleward expansion site after arriving at the onset arc and before poleward expansion. We also could not identify another preonset 


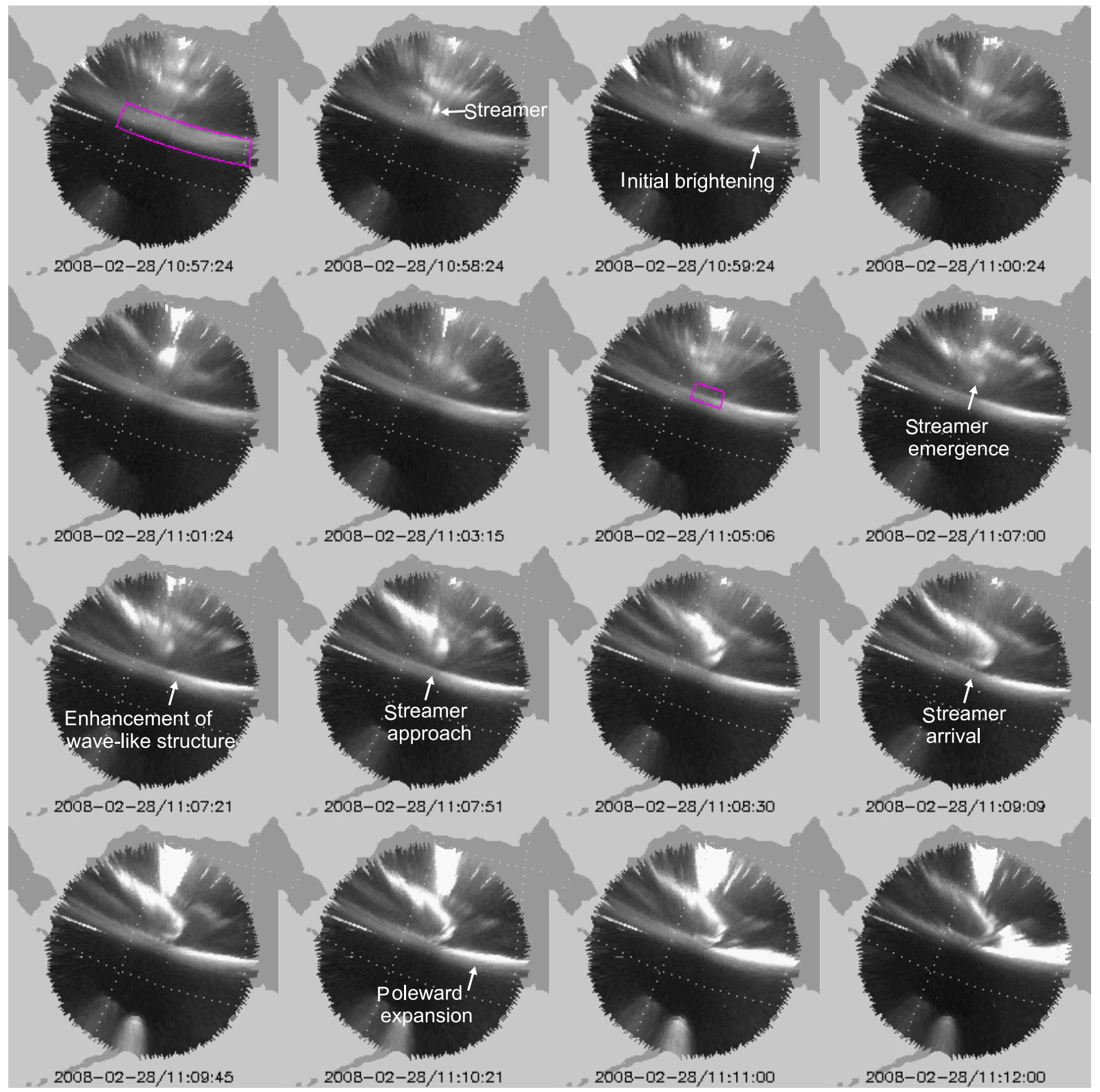

Figure 5. Selected auroral images obtained from the THEMIS GBO all-sky imager at MCGR between 10:57 and 11:12 UT on 28 February 2008. The maximum count value of the black-white scale is 3000 , except that it is 4000 for the last image.

aurora moving along the onset arc from the outside of the field of view toward the initial brightening site.

Nishimura et al. (2011) seem to have taken the arrival time of the streamer as the time of poleward expansion (11:10 UT), which is $\sim 2$ or 1 min later than our determination. In any case, the arrival time was later than the times of initial brightening and enhancement of the wave-like structure.

\subsection{March 2008 event}

According to Nishimura et al.'s $(2010,2011)$ list, this event was an isolated one without the subsequent significant poleward expansion. Figure 8 shows selected ASI images from GILL (see also Supplement Movie S3). The average count variations along the auroral onset arc (the magenta boxes in the 06:01:33 and 06:02:33 UT panels of Fig. 8) are shown in Fig. 9. The luminosity was almost constant before initial brightening in this event, unlike the other events shown above. Lessard et al. (2007) showed an event in which only $630.0 \mathrm{~nm}$ emission was intensified while 557.7 and $486.1 \mathrm{~nm}$ 

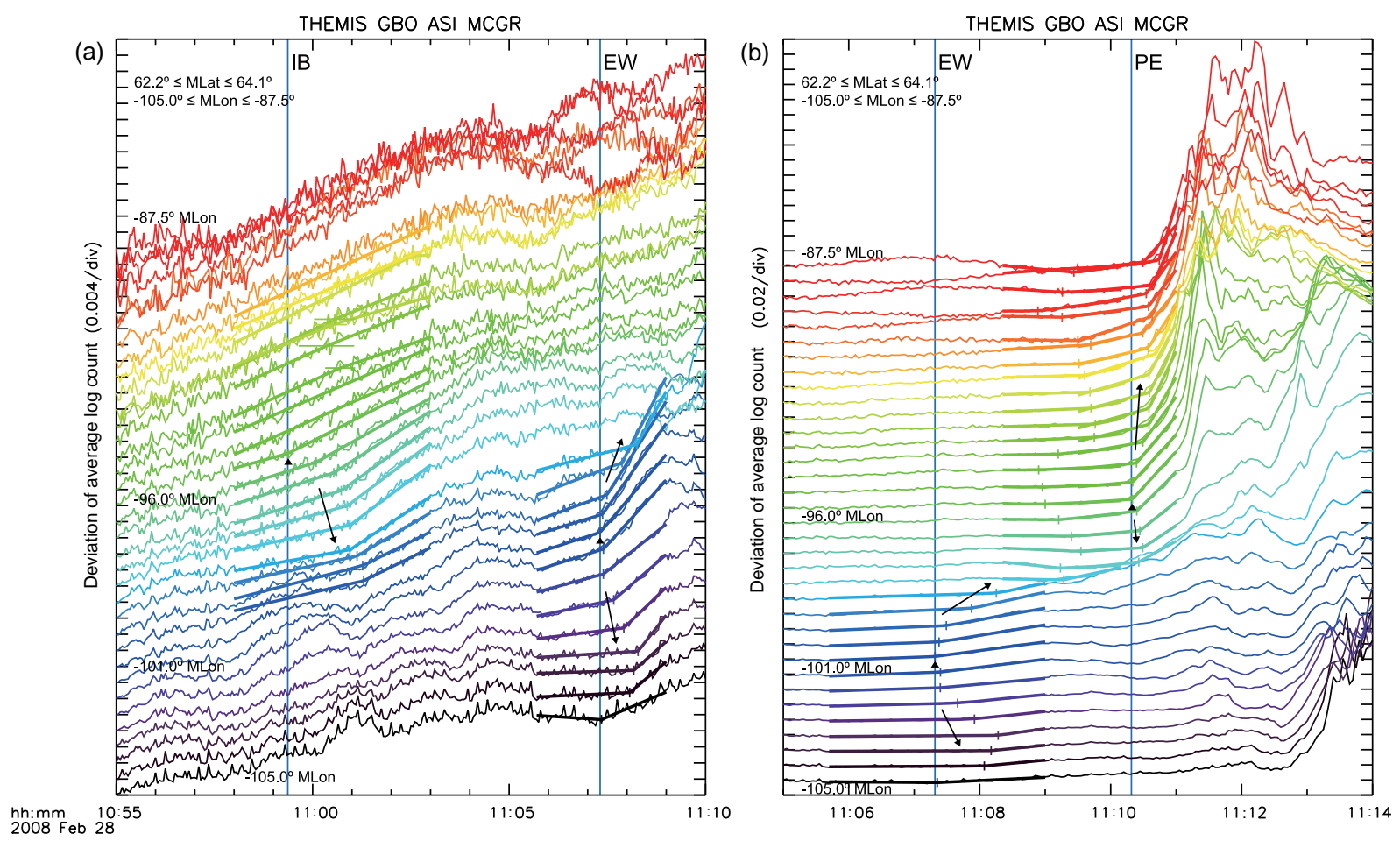

Figure 6. Variations of the averages of the logarithm of count for areas of $0.5^{\circ}$ longitudinal width in the region indicated in the top of this figure, or the magenta box in the 10:57:24 UT panel of Fig. 5, including the auroral onset arc obtained from the THEMIS GBO all-sky imager at MCGR on 28 February 2008. The format is the same as Fig. 3.

Table 2. UT of auroral onset arc development and preonset aurora, and the arrival location of the preonset aurora relative to the initial brightening or poleward expansion site $(\Delta \mathrm{MLT})$ for the 28 February 2008 substorm event.

\begin{tabular}{lll}
\hline 28 Feb 2008 & The present study & Previous studies \\
\hline Auroral onset arc development & & \\
Initial brightening & $10: 59: 22$ & \\
Enhancement of the wave-like structure & $11: 07: 19$ & \\
Poleward expansion & $11: 10: 19$ & \multirow{2}{*}{ 11:10 (Nishimura et al., 2010, 2011) } \\
\hline Preonset aurora & & \\
Emergence & $\sim 11: 07$ & \\
Arrival just poleward of/at the onset arc & $11: 07: 50 / 11: 09: 09$ & \multirow{2}{*}{$11: 10 ?$ (Nishimura et al., 2010, 2011) } \\
MMLT, h & $\sim 0.5$, west & \\
\hline
\end{tabular}

emissions were unchanged. We surmise that this was the case with our event shown here. Since $557.7 \mathrm{~nm}$ emission is often more intense than 630.0 and $486.1 \mathrm{~nm}$ emissions, the whitelight images from THEMIS ASI possibly reflected the unchanged $557.7 \mathrm{~nm}$ emission in the present event. Then initial brightening occurred near the middle of the field of view at $\sim 67^{\circ}$ magnetic latitude and $\sim 0 \mathrm{~h}$ MLT at 06:02:15 UT. Figure 9a shows that the breakpoint due to slight increase in the trend appeared first at a localized segment (on a green line) at this time and then appeared at the westward and eastward segments (on neighbors) successively. That is, the onset arc gradually grew brighter and extended westward and eastward. Figure 9a also shows that the luminosity oscil- lated after initial brightening with increasing the trend, due to gradual growth of the wave-like structure of the auroral onset arc. At 06:03:15 UT, 1 min after initial brightening, the onset arc as well as the wave-like structure were further enhanced somewhat eastward of the initial brightening site. The breakpoint due to larger increase appeared in this part of the onset arc (on a yellow green line and then mainly on western neighbors) in Fig. 9a. At 06:06:04 UT, another $\sim 3$ min later, poleward expansion began to the east of the initial brightening site. The breakpoint due to explosive increase appeared first on green lines and then on neighbors in Fig. 9b. The times of auroral onset arc development are summarized in Table 3. 


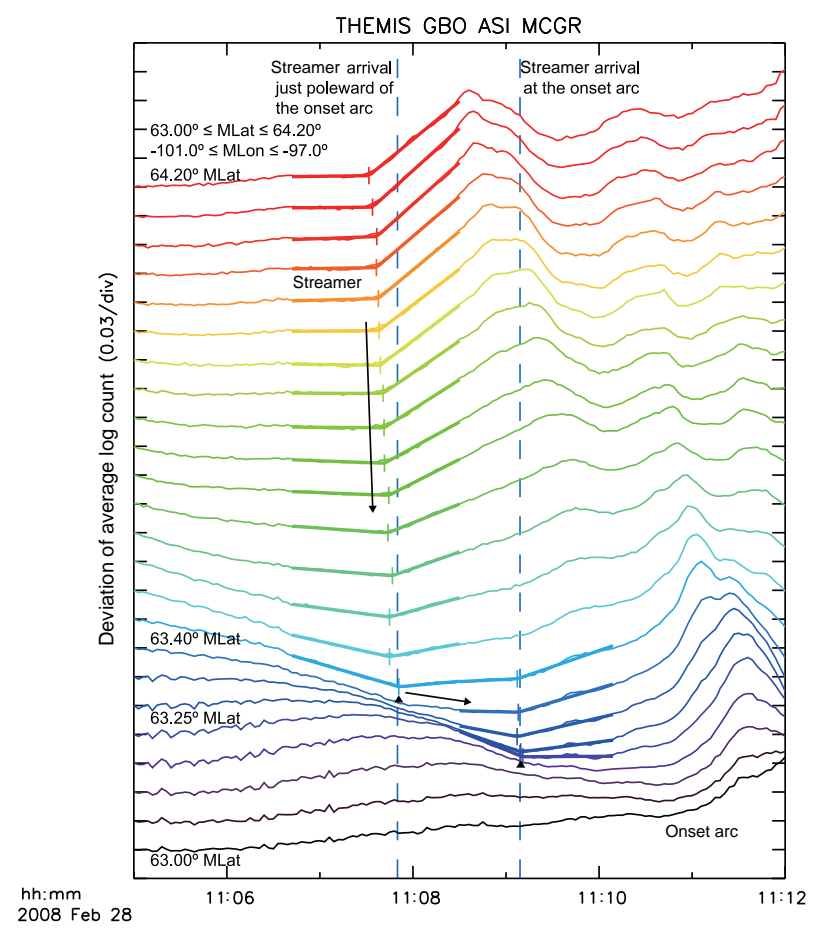

Figure 7. Variations of the averages of the logarithm of count for areas of $0.05^{\circ}$ latitudinal width in the region indicated in the top of this figure, or the magenta box in the 11:05:06 UT panel of Fig. 5, including the path of the equatorward moving auroral streamer obtained from the THEMIS GBO all-sky imager at MCGR on 25 February 2008. The format is the same as Fig. 4.

Nishimura et al. (2010, 2011), Liu et al. (2008), and Rae et al. (2012, 2017) analyzed this event and determined the breakup arc formation time as 06:04 UT. Nishimura et al. (2016) revised the times of auroral development, determining the initial brightening time as 06:03:25 UT and the poleward expansion time as 06:06:18 UT. The breakup arc formation and initial brightening times determined by these previous studies nearly agree with $\sim 1 \mathrm{~min}$ after our time of enhancement of the wave-like structure, rather than our initial brightening time. This is possibly because they determined the time from substantial intensification, while we paid attention to initial faint aurora as well. The poleward expansion time of Nishimura et al. (2016) roughly agrees with our determination.

For this event, we could not find any preonset auroras propagating from the auroral poleward boundary to the onset arc, as also pointed out by Rae et al. (2017). A bright east-west-aligned arc appeared at $\sim 05: 53 \mathrm{UT}$ at a few degrees higher latitude than the onset arc. Another bright eastwest-aligned arc appeared further poleward of this arc at 06:00:05 UT (green to red lines of auroral count variations in Fig. 10a). These two arcs did not approach the onset arc. Furthermore, a faint east-west-aligned arc appeared between the prior arc and the onset arc at 05:59:43 UT (blue lines in
Fig. 10a). Although it slightly moved equatorward, it stopped just poleward of the onset arc soon. In any case, this faint arc was not the preonset aurora propagating from the poleward boundary as proposed by Nishimura et al. (2010). It is not clear which poleward auroral arc Nishimura et al. (2010, 2011) identified as the preonset aurora, but they seem to have mistakenly counted the aurora unrelated to auroral onset arc development associated with substorm.

\section{Discussion}

\subsection{Effect of flow and flow vortices}

In the previous section we showed the relative timings of the three steps of auroral onset arc development and arrival of the auroral streamers at the onset arc on the basis of ASI data. As we mentioned in the introduction and as depicted in Fig. 1, we should also pay attention to the effect of flow and flow vortices related to the auroral streamer, which can be observed by ground-based radars and magnetometers, not by ASIs. We checked the SuperDARN data (Greenwald et al., 1995), but unfortunately there were too few echoes to identify the flow vortices and their spatial scale. Ground magnetometer data were also available, but the ground stations were too sparsely distributed to identify the small-scale flow vortices associated with the auroral streamer for the present events. Hence we just discuss the flow vortex effect for the first two streamer events shown in Sects. 3.1 and 3.2 by considering that a typical spatial scale of the vortex is $\sim 1 \mathrm{~h}$ in MLT.

For the 25 February 2008 event shown in Sect. 3.1, the separation of the arrival point of the auroral streamer and the sites of auroral onset arc development was $\sim 0.3 \mathrm{~h}$ in MLT, which may be within the expected eastern flow vortex scale. (There were too few SuperDARN echoes on the eastern side of the auroral streamer to identify the eastern flow vortex and its spatial scale, while the western flow vortex was possibly identified to have a spatial scale of $\sim 1 \mathrm{~h}$ in MLT (not shown).) However, the auroral diffuse patch was still poleward of the onset arc at initial brightening, and the auroral streamer appeared after initial brightening, as shown above. Taking the equatorward direction of the diffuse patch and the expected flow vortex scale into account (Fig. 1a), the central part of the flow and the flow vortices should have been still poleward of the onset arc at initial brightening as well. Hence it is unlikely that they directly affected initial brightening. Thus, even if the flow vortex is considered, we can conclude that the present results are inconsistent with Nishimura et al.'s (2010) scenario in which the preonset aurora should reach the initial brightening site before initial brightening. Here we cannot deny the possibility that the auroral streamer affected enhancement of the wave-like structure and the subsequent poleward expansion, since the corresponding eastern flow vortex may contact the site of enhancement of the wave- 


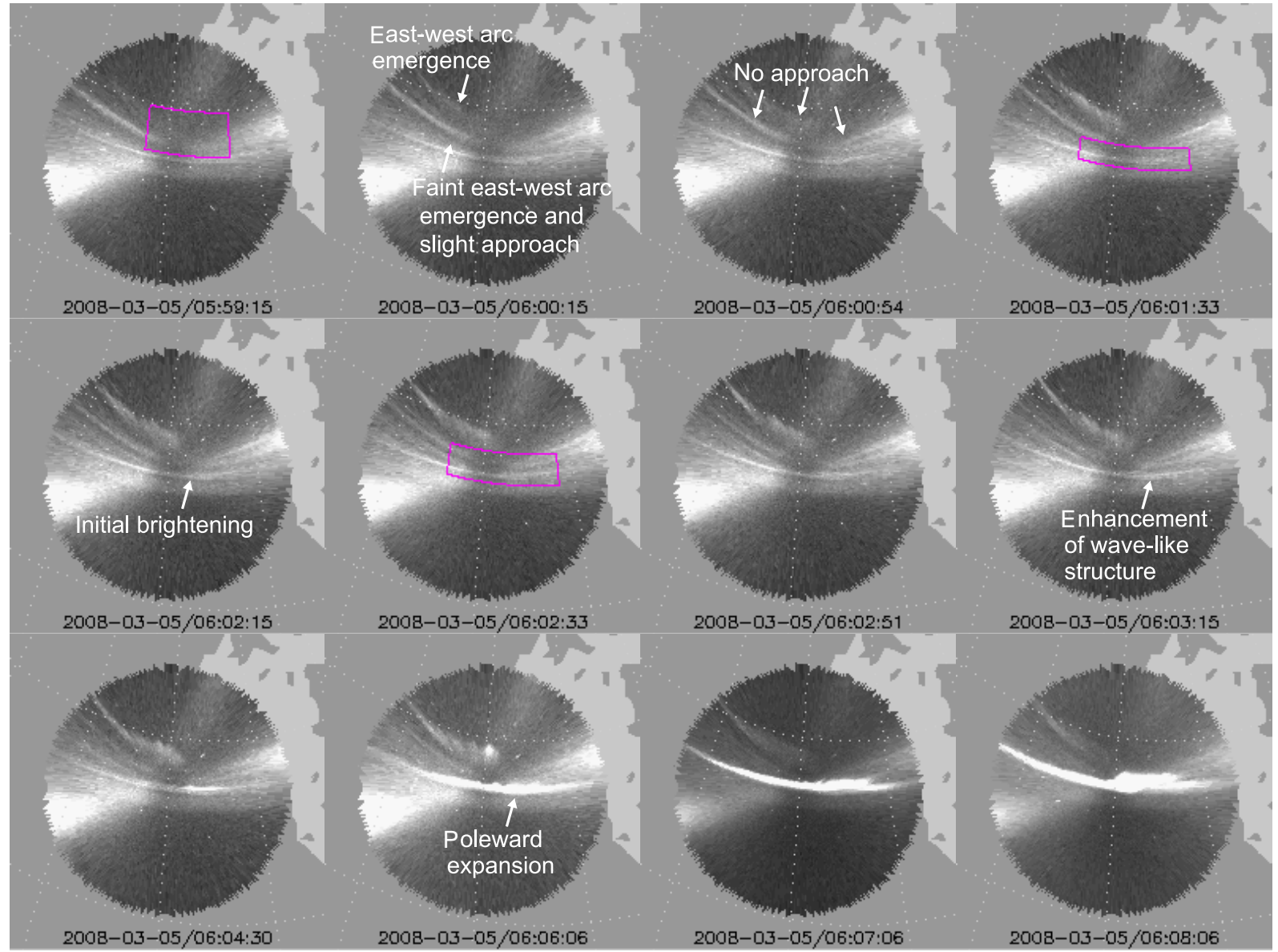

Figure 8. Selected auroral images obtained from the THEMIS GBO all-sky imager at GILL between 05:59 and 06:09 UT on 5 March 2008. The maximum count values of the black-white scale are 1000 for 05:59:15 to 06:06:06 UT and 2000 for the later times.

Table 3. UT of auroral onset arc development and preonset aurora for the 5 March 2008 substorm event.

\begin{tabular}{lll}
\hline 5 Mar 2008 & The present study & Previous studies \\
\hline $\begin{array}{l}\text { Auroral onset arc development } \\
\text { Initial brightening }\end{array}$ & $06: 02: 15$ & \\
Enhancement of the wave-like structure & $06: 03: 15$ & $06: 03: 25$ (Nishimura et al., 2016) \\
& & $06: 04$ (Nishimura et al., 2010, 2011) \\
& & $06: 04$ (Liu et al., 2008) \\
& $06: 04$ (Rae et al., 2012, 2017) \\
Poleward expansion & $06: 06: 04$ & $06: 06: 18$ (Nishimura et al., 2016) \\
\hline Preonset aurora & $\sim 05: 53,06: 00: 05$ & \\
Emergence & arrived (Nishimura et al., 2010, 2011) \\
Arrival just poleward of/at the onset arc & not arrived &
\end{tabular}

like structure and poleward expansion before these auroral developments, but there are insufficient data to confirm this.

For the 28 February 2008 event shown in Sect. 3.2, the separation of the arrival point of the auroral streamer and the sites of auroral onset arc development was $\sim 0.5 \mathrm{~h}$ or less in MLT, probably within the expected eastern flow vortex scale, although the auroral streamer reached near the onset arc be- tween the times of enhancement of the wave-like structure and poleward expansion. (There were too few SuperDARN echoes on either side of the auroral streamer to identify a flow vortex.) Considering the direction of the auroral streamer and the expected flow vortex scale, the auroral streamer was directed equatorward and eastward and was still poleward of the onset arc at enhancement of the wave-like structure. Since 

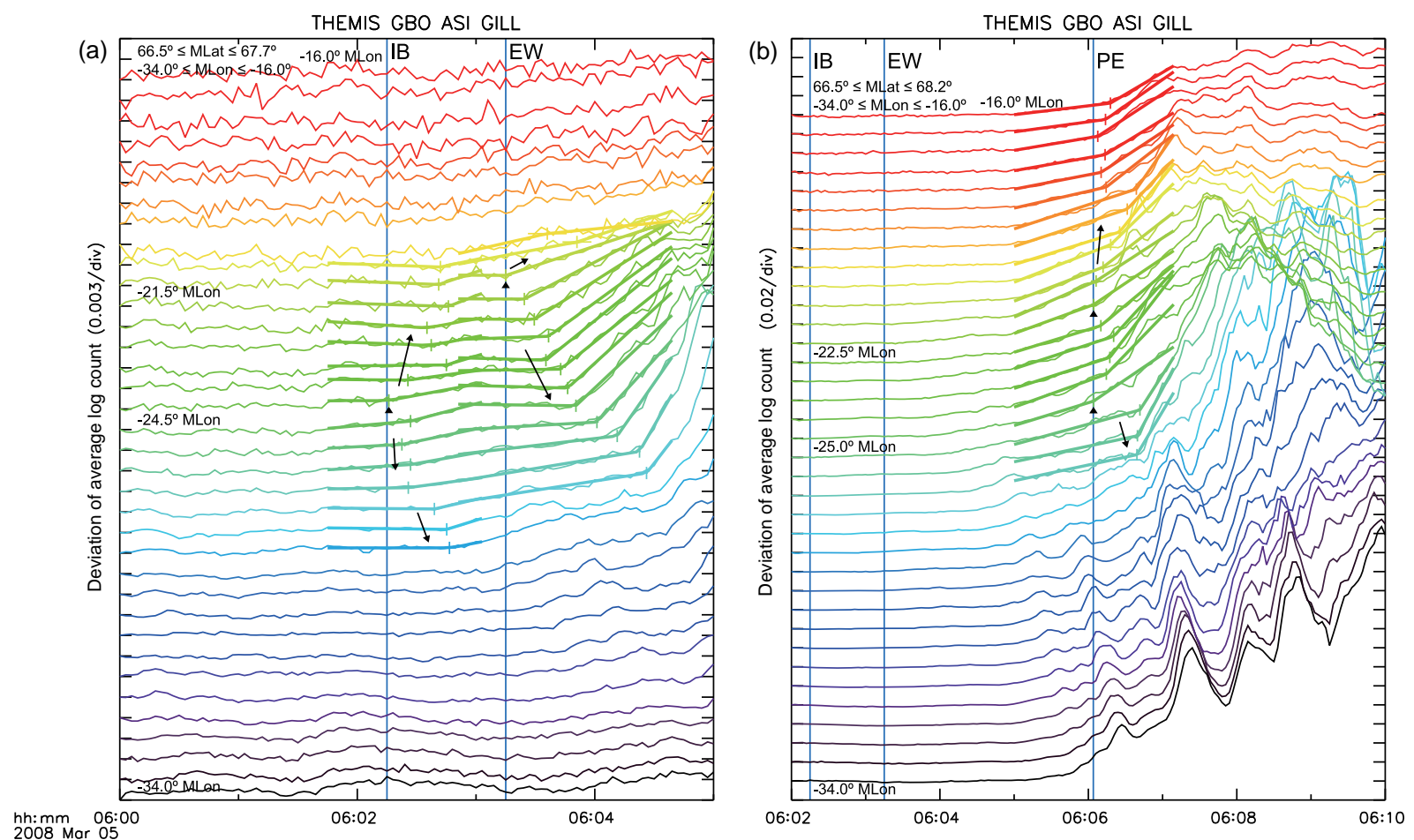

Figure 9. Variations of the averages of the logarithm of count for areas of $0.5^{\circ}$ longitudinal width in the region indicated in the top of this figure, or the magenta boxes in the (a) 06:01:33 and (b) 06:02:33 UT panels of Fig. 8, including the auroral onset arc obtained from the THEMIS GBO all-sky imager at GILL on 5 March 2008. The format is the same as Fig. 3.

the central part of the flow and the eastern flow vortex should have been poleward of the onset arc as well (Fig. 1c), it is unlikely that the flow and the eastern flow vortex affected the onset arc at this time. The auroral streamer then turned in the equatorward and westward direction at $\sim 11: 08 \mathrm{UT}$ $(\sim 1$ min after enhancement of the wave-like structure, but $\sim 2$ min before poleward expansion), so the eastern flow vortex may have contacted the poleward expansion site just before the auroral streamer arrived at the onset arc (Fig. 1b). That is, there may be a possibility that the eastern flow vortex affected poleward expansion. Nevertheless, the appearance timing indicates that this auroral streamer cannot be related to initial brightening, which is inconsistent with Nishimura et al.'s (2010) scenario.

\subsection{Summary and open questions}

In the present paper, based on THEMIS GBO ASI data, we revisited three substorm events, which Nishimura et al. $(2010,2011)$ identified as being triggered by the processes associated with preonset auroras. Unlike most previous studies that determined only one or two timings of auroral onset arc development, we attempted to determine the three timings of initial brightening, enhancement of the wave-like structure, and poleward expansion to make our discussion about the arrival timing of the preonset aurora relative to the three auroral steps and the role of the preonset aurora in the auroral steps clearer. Here we adopted a more quantitative method for timing determination, paying attention to not only substantial intensification but also initial faint auroras. We also discussed the spatial relationship between the auroral onset arc, the auroral streamer, and the associated flow channel and flow vortices.

Our more detailed analysis showed that preonset auroral streamers reached the auroral onset arc but away from the initial brightening site after initial brightening for two events, while no preonset aurora reaching the initial brightening site could be identified for the other event. This result suggests that the processes associated with the auroral streamers are unlikely to affect at least initial brightening, even if we consider not only the presence and arrival timing and location of the auroral streamers but also the scale of the corresponding flow and flow vortices. Although we examined only the three events, these results possibly suggest that the preonset aurora scenario is questionable, and at least the processes associated with the preonset aurora are not necessary conditions for initial brightening. Murphy et al. (2014b), for example, reached the same conclusion. Furthermore, Nishimura et al. (2010) showed that not all events were accompanied by preonset auroras; this result itself implies that conclusion. Thus careful, detailed reexamination of other Nishimura et al. $(2010,2011)$ events is required to prove or disprove the preonset aurora scenario in future studies. 

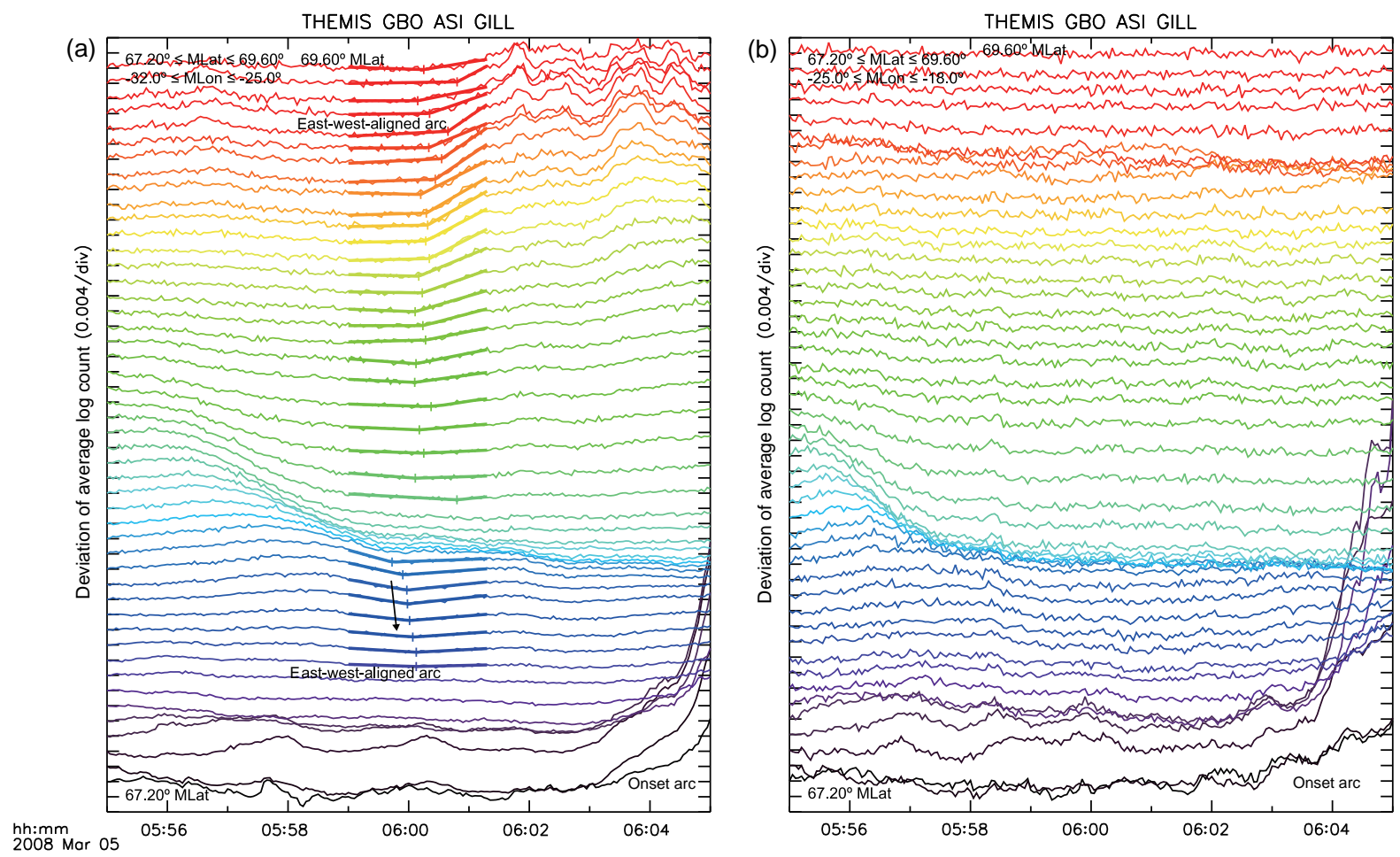

Figure 10. Variations of the averages of the logarithm of count for areas of $0.05^{\circ}$ latitudinal width in the region indicated in the top of this figure, or the (a) western and (b) eastern halves of the magenta box in the 05:59:15 UT panel of Fig. 8, including the two east-west arcs obtained from the THEMIS GBO all-sky imager at GILL on 5 March 2008. The format is the same as Fig. 4.

For testing the preonset aurora scenario further in future studies, we list a series of open questions that arose from the present and previous studies. We suggest the issues not only from the viewpoint of existence or nonexistence of the preonset aurora but also from different viewpoints.

1. First of all, it is necessary to reexamine the relative timings of initial auroral brightening and arrival of the preonset aurora or the corresponding flow and flow vortex at the site of auroral onset arc development carefully and exactly, paying attention to faint auroras as well. Furthermore, to clarify the causal relationship, the timings of enhancement of the wave-like auroral structure and poleward expansion should be determined as well. As shown above, the auroral streamer arrived at the auroral onset arc after, not before, initial brightening, i.e., between initial brightening and enhancement of the wavelike structure for the present first event and between enhancement of the wave-like structure and poleward expansion for the second event. Hence it should be reexamined whether a preonset aurora or the corresponding flow and flow vortex really arrived at the onset arc before initial brightening for other events of Nishimura et al. (2010, 2011). It should also be clarified what role the preonset aurora plays in onset arc development.
2. We should examine how far from the initial brightening site the path and final arrival point of each preonset aurora are. In the present study, the final arrival point was $\sim 0.3-0.5 \mathrm{~h}$ in MLT away from the initial brightening or poleward expansion site for the first and second events. The auroral streamers did not move toward the initial brightening site after they reached the auroral onset arc, although Nishimura et al. (2010) proposed that the preonset aurora reaches the initial brightening site. Here, as mentioned in the introduction, we should consider the spatial scales of the central part of the flow and the flow vortices. There may be a possibility that the central part of the flow or the flow vortex affects the auroral onset arc if the auroral streamer is separated by less than $\sim 1 \mathrm{~h}$ in MLT. We should study, however, whether the effect of the flow vortex can really lead to initial auroral brightening.

3. There are a few types of preonset aurora, but is it valid to mix them? Mende et al. (2011) showed that the preonset aurora events identified by Nishimura et al. (2010) can be categorized as having a north-south-aligned equatorward moving streamer, an east-west-aligned equatorward moving arc, or no equatorward moving aurora at longitudes of initial brightening. Hence it should be validated whether these types can be regarded as being the same phenomenon in spite of different appearances. 


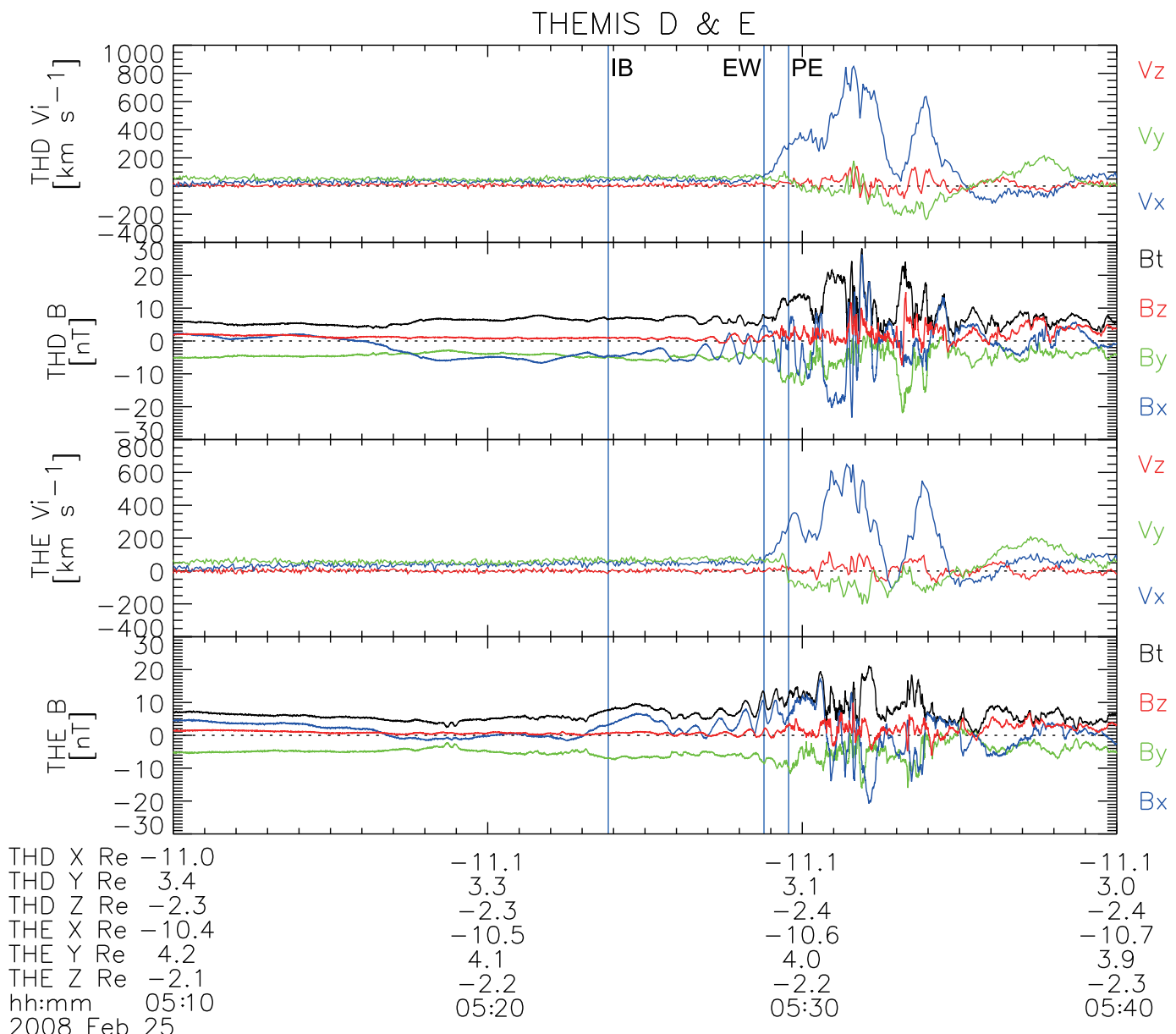

Figure 11. The $3 \mathrm{~s}$ resolution ion velocity and the $0.25 \mathrm{~s}$ resolution magnetic field in GSM coordinates obtained from the electrostatic analyzer (ESA) (McFadden et al., 2008) and solid state telescope (SST) (Angelopoulos, 2008) and from the fluxgate magnetometer (FGM) (Auster et al., 2008), respectively, onboard THEMIS D (THD) and E (THE) from 05:10 to 05:40 UT on 25 February 2008. The left-hand, middle, and right-hand vertical lines indicate the times of initial auroral brightening (IB), enhancement of the wave-like structure (EW), and poleward expansion (PE), respectively.

4. It should also be proved whether both north-south and east-west auroras correspond to fast earthward flows in the magnetotail and whether the width, direction, and position of the preonset auroras are consistent with those of fast earthward flows in the magnetotail. Regarding the direction of the fast earthward flow in the near-Earth magnetotail, for the 25 February 2008 event described in Sect. 3.1, THEMIS D, located in the plasma sheet at $(X, Y) \sim(-11.1,3.1) R_{\mathrm{E}}$, observed a fast earthward flow at 05:28:20 UT, $\sim 4.5$ min after initial brightening and $\sim 30 \mathrm{~s}$ before enhancement of the wave-like auroral structure (Fig. 11). THEMIS E, located in the plasma sheet at $(X, Y) \sim(-10.6,4.0)$ $R_{\mathrm{E}}$ or $\sim 1 R_{\mathrm{E}}$ earthward and duskward of THEMIS $\mathrm{D}$, also observed a fast earthward flow at 05:28:42 UT, $\sim 20 \mathrm{~s}$ after the THEMIS D observation and just before enhancement of the wave-like structure. These fast flows had a small duskward component in the front part.
Hence the fast flow propagated from the tailward region in the earthward and slightly duskward direction. If this observed magnetotail fast flow is mapped to the ionosphere and unless the magnetic field line is extremely distorted by a field-aligned current, the auroral streamer should have moved southwestward. This expected direction, however, seems to be inconsistent with that of the observed auroral streamer and diffuse patch, which propagated southeastward and nearly equatorward, respectively, in the premidnight sector. If the observed auroral streamer and diffuse patch are mapped to the magnetic equator in the magnetotail, the magnetotail flow should be directed earthward and dawnward. Hence it is possibly questionable whether the auroral streamer and diffuse patch really correspond to the fast earthward flow observed by THEMIS D and E. Xing et al. (2010) also examined earthward flows in the magnetotail corresponding to preonset streamers. Considering the dawn- 
dusk component together, however, not all magnetotail flows seem to be consistent with the propagation direction of the streamers (see their Figs. 2 and 9).

5. In addition, determining the origin of the fast earthward flow in the magnetotail is a clue. If the auroral streamer appears at the auroral poleward boundary and between the auroral poleward and equatorward boundaries, the corresponding magnetotail flow should have originated at the distant neutral line at $X \sim-130 R_{\mathrm{E}}$ and at the near-Earth neutral line at $X \sim-20 R_{\mathrm{E}}$, respectively, on average. Some previous studies attempted to determine the location and timing of magnetic reconnection from flow or beam observations in the magnetotail (e.g., Elphic et al., 1995; Kazama and Mukai, 2003; Nagata et al., 2006; Nishimura et al., 2013b), but it seems challenging to determine them without spatial and temporal ambiguity.

6. There is a possibility that some preonset auroras are too weak to be observed by THEMIS imagers. As shown by Kepko et al. (2009), high-sensitivity imagers for $630.0 \mathrm{~nm}$ emission may be able to detect weak auroral streamers or patches moving toward the auroral onset arc. Otherwise, it may be possible that particle precipitation is too weak to cause any aurora. In this case, ionospheric flow observations are needed to detect streamers.

7. Nishimura et al.'s $(2010,2011)$ event list included events without significant poleward expansion, i.e., pseudobreakups. They did not distinguish between pseudobreakups and fully fledged substorms in their analysis. If the preonset aurora scenario mentions not only initial brightening but also later enhancement of the wave-like structure and poleward expansion, pseudobreakup events should be separated in discussing the role of the preonset aurora in auroral onset arc development. Furthermore, according to Frey (2010), some events of Nishimura et al. (2010) were mistakenly identified as substorm events and should be categorized as pseudobreakups or substorm intensifications. Hence it would be necessary to reexamine the category of each of Nishimura et al.'s (2010, 2011) events.

8. It should be discussed whether the arrival time of the fast earthward flow in the near-Earth magnetotail corresponding to the preonset aurora is consistent with the growth time of an instability leading to initial auroral brightening. The preonset aurora scenario suggests that the fast earthward flow evolves an onset instability, such as ballooning instability, leading to initial auroral brightening (Nishimura et al., 2014). Ballooning mode waves may be excited a few minutes before initial brightening (as determined with a spacecraft-borne auroral imager) (Saito et al., 2008). The time difference between the arrival of the preonset aurora at the onset arc and initial brightening that Nishimura et al. (2010) showed seems consistent with the growth time of an instability. As mentioned above, however, the relative timing of streamer arrival and initial brightening need to be reexamined, so this test should be done on the basis of the revised timings.

9. Finally, even if the processes associated with a preonset aurora are not necessary conditions for initial brightening, it may still be possible that for some substorms, a preonset aurora and the corresponding fast earthward flow in the magnetotail make a seed of substorm instabilities leading to initial auroral brightening, magnetic reconnection or current disruption/dipolarization, and later auroral development (enhancement of the wavelike structure and poleward expansion) and magnetotail development, as mentioned in the discussion of the first and second events of the present study. Testing this possibility may be important for full understanding of substorm development.

Data availability. The THEMIS GBO ASI and spacecraft data are available at the Space Sciences Laboratory, University of California, Berkeley (http://themis.ssl.berkeley.edu/, Space Science Laboratory, 2018). The Dst and AE indices are available at the World Data Center for Geomagnetism, Kyoto (http://wdc.kugi.kyoto-u.ac. jp/, World Data Center for Geomagnetism, 2018). The SuperDARN data are available at http://vt.superdarn.org/ (Virginia Polytechnic Institute and State University, 2018) and http://ergsc.isee.nagoya-u. ac.jp/ (ERG Science Center, 2018).

Supplement. The supplement related to this article is available online at: https://doi.org/10.5194/angeo-36-1419-2018-supplement.

Author contributions. YM carried out the analysis and prepared the manuscript. AI participated in discussing the results, read the manuscript, and commented on it.

Competing interests. The authors declare that they have no conflict of interest.

Acknowledgements. One of the authors (Yukinaga Miyashita) thanks Yukitoshi Nishimura, Kyle Murphy, Emma Spanswick, and Jian Yang for inviting him to present the early results of this paper at a focus group at GEM summer and mini workshops in 2015. The present study was in part performed when Yukinaga Miyashita worked at the University of California, Los Angeles, and the Institute for Space-Earth Environmental Research, Nagoya University. Yukinaga Miyashita thanks Vassilis Angelopoulos and Shinobu Machida for their support and useful comments. This work was supported by a Grant-in-Aid for Scientific Research (26247082) 
and Program for Advancing Strategic International Networks to Accelerate the Circulation of Talented Researchers (G2602) of the Japan Society for the Promotion of Science. We acknowledge NASA contract NAS5-02099 and Vassilis Angelopoulos for use of data from the THEMIS mission. We thank Stephen B. Mende and Eric Donovan for use of the THEMIS GBO ASI data, the Canadian Space Agency (CSA) for logistical support in fielding and data retrieval from the GBO stations, and the National Science Foundation (NSF) for support of GIMNAST through grant AGS-1004736. We thank Karl-Heinz Glassmeier, Hans-Ulrich Auster, and Wolfgang Baumjohann for use of the THEMIS FGM data provided under the lead of the Technical University of Braunschweig and with financial support through the German Ministry for Economy and Technology and the German Center for Aviation and Space (DLR) under contract 50 OC 0302. We thank Charles W. Carlson and James P. McFadden for use of the THEMIS ESA data and Davin Larson and Robert P. Lin for use of the THEMIS SST data. We thank the World Data Center for Geomagnetism, Kyoto, for the Dst and AE indices. We thank Virginia Polytechnic Institute and State University and the ERG Science Center for the SuperDARN data. The ERG Science Center is operated by the Institute of Space and Astronautical Science, Japan Aerospace Exploration Agency, and Institute for Space-Earth Environmental Research, Nagoya University.

Edited by: Christopher Owen

Reviewed by: two anonymous referees

\section{References}

Akasofu, S.-I.: The development of the auroral substorm, Planet. Space Sci., 12, 273-282, https://doi.org/10.1016/00320633(64)90151-5, 1964.

Amm, O., Pajunpää, A., and Brandström, U.: Spatial distribution of conductances and currents associated with a north-south auroral form during a multiple-substorm period, Ann. Geophys., 17, 1385-1396, https://doi.org/10.1007/s00585-999-1385-6, 1999.

Angelopoulos, V.: The THEMIS mission, Space Sci. Rev., 141, 534, https://doi.org/10.1007/s11214-008-9336-1, 2008.

Angelopoulos, V., McFadden, J. P., Larson, D., Carlson, C. W., Mende, S. B., Frey, H., Phan, T., Sibeck, D. G., Glassmeier, K.-H., Auster, U., Donovan, E., Mann, I. R., Rae, I. J., Russell, C. T., Runov, A., Zhou, X.-Z., and Kepko, L.: Tail reconnection triggering substorm onset, Science, 321, 931-935, https://doi.org/10.1126/science.1160495, 2008.

Auster, H. U., Glassmeier, K. H., Magnes, W., Aydogar, O., Baumjohann, W., Constantinescu, D., Fischer, D., Fornacon, K. H., Georgescu, E., Harvey, P., Hillenmaier, O., Kroth, R., Ludlam, M., Narita, Y., Nakamura, R., Okrafka, K., Plaschke, F., Richter, I., Schwarzl, H., Stoll, B., Valavanoglou, A., and Wiedemann, M.: The THEMIS fluxgate magnetometer, Space Sci. Rev., 141, 235-264, https://doi.org/10.1007/s11214-008-9365-9, 2008.

Baker, D. N., Pulkkinen, T. I., Angelopoulos, V., Baumjohann, W., and McPherron, R. L.: Neutral line model of substorms: Past results and present view, J. Geophys. Res., 101, 12975-13010, https://doi.org/10.1029/95JA03753, 1996.
Chang, T.-F. and Cheng C.-Z.: Relationship between wavelike auroral arcs and Pi2 disturbances in plasma sheet prior to substorm onset, Earth Planet. Space, 67, 168, https://doi.org/10.1186/s40623-015-0334-8, 2015.

Chang, T. F., Cheng, C. Z., Chiang, C. Y., and Chen, A. B.: Behavior of substorm auroral arcs and $\mathrm{Pi} 2$ waves: implication for the kinetic ballooning instability, Ann. Geophys., 30, 911-926, https://doi.org/10.5194/angeo-30-911-2012, 2012.

Chaston, C. C., Bonnell, J. W., Peticolas, L. M., Carlson, C. W., McFadden, J. P., and Ergun, R. E.: Driven Alfven waves and electron acceleration: A FAST case study, Geophys. Res. Lett., 29, 1535, https://doi.org/10.1029/2001GL013842, 2002.

Coxon, J. C., Rae, I. J., Forsyth, C., Jackman, C. M., Fear, R. C., and Anderson, B. J.: Birkeland currents during substorms: Statistical evidence for intensification of Regions 1 and 2 currents after onset and a localized signature of auroral dimming, J. Geophys. Res.-Space, 122, 6455-6468, https://doi.org/10.1002/2017JA023967, 2017.

Davis, T. N.: The morphology of the auroral displays of 1957-1958: 2. Detail analyses of Alaska data and analyses of high-latitude data, J. Geophys. Res., 67, 75-110, https://doi.org/10.1029/JZ067i001p00075, 1962.

Deehr, C. and Lummerzheim, D.: Ground-based optical observations of hydrogen emission in the auroral substorm, J. Geophys. Res., 106, 33-44, https://doi.org/10.1029/2000JA002010, 2001.

Donovan, E., Mende, S., Jackel, B., Frey, H. Syrjäsuo, M., Voronkov, I., Trondsen, T., Peticolas, L., Angelopoulos, V., Harris, S., Greffen, M., and Connors, M.: The THEMIS allsky imaging array - system design and initial results from the prototype imager, J. Atmos. Sol.-Terr. Phy., 68, 1472-1487, https://doi.org/10.1016/j.jastp.2005.03.027, 2006.

Donovan, E., Mende, S., Jackel, B., Syrjäsuo, M., Meurant, M., Voronkov, I., Frey, H. U., Angelopoulos, V., and Connors, M.: The azimuthal evolution of the substorm expansive phase onset aurora, in: Proceedings of International Conference on Substorms-8, edited by: Syrjäsuo, M. and Donovan, E., University of Calgary, Alberta, Canada, 55-60, 2007.

Elphic, R. C., Onsager, T. G., Thomsen, M. F., and Gosling, J. T.: Nature and location of the source of plasma sheet boundary layer ion beams, J. Geophys. Res., 100, 1857-1869, https://doi.org/10.1029/94JA02419, 1995.

Elphinstone, R. D., Hearn, D. J., Cogger, L. L., Murphree, J. S., Singer, H., Sergeev, V., Mursula, K., Klumpar, D. M., Reeves, G. D., Johnson, M., Ohtani, S., Potemra, T. A., Sandahl, I., Nielsen, E., Persson, M., Opgenoorth, H., Newell, P. T., and Feldstein, Y. I.: Observations in the vicinity of substorm onset: Implications for the substorm process, J. Geophys. Res., 100, 7937-7969, https://doi.org/10.1029/94JA02938, 1995.

The ERG Science Center: available at: http://ergsc.isee.nagoya-u. ac.jp/ last access: 17 October 2018.

Frey, H. U.: Comment on "Substorm triggering by new plasma intrusion: THEMIS all-sky imager observations" by Y. Nishimura et al., J. Geophys. Res., 115, A12232, https://doi.org/10.1029/2010JA016113, 2010.

Friedrich, E., Samson, J. C., and Voronkov, I.: Ground-based observations and plasma instabilities in auroral substorms, Phys. Plasmas, 8, 1104-1110, https://doi.org/10.1063/1.1355678, 2001.

Gabrielse, C., Angelopoulos, V., Runov, A., Frey, H. U., McFadden, J., Larson, D. E., Glassmeier, K.-H., Mende, S., Rus- 
sell, C. T., Apatenkov, S., Murphy, K. R., and Rae, I. J.: Timing and localization of near-Earth tail and ionospheric signatures during a substorm onset, J. Geophys. Res., 114, A00C13, https://doi.org/10.1029/2008JA013583, 2009.

Greenwald, R. A., Baker, K. B., Dudeney, J. R., Pinnock, M., Jones, T. B., Thomas, E. C., Villain, J.-P., Cerisier, J.-C., Senior, C., Hanuise, C., Hunsucker, R. D., Sofko, G., Koehler, J., Nielsen, E., Pellinen, R., Walker, A. D. M., Sato, N., and Yamagishi, H.: DARN/SuperDARN: A global view of the dynamics of high-latitude convection, Space Sci. Rev., 71, 761-796, https://doi.org/10.1007/BF00751350, 1995.

Henderson, M. G.: Observational evidence for an inside-out substorm onset scenario, Ann. Geophys., 27, 2129-2140, https://doi.org/10.5194/angeo-27-2129-2009, 2009.

Imber, S. M., Slavin, J. A., Auster, H. U., and Angelopoulos, V.: A THEMIS survey of flux ropes and traveling compression regions: Location of the near-Earth reconnection site during solar minimum, J. Geophys. Res.-Space, 116, A02201, https://doi.org/10.1029/2010JA016026, 2011.

Kalmoni, N. M. E., Rae, I. J., Watt, C. E. J., Murphy, K. R., Forsyth, C., and Owen, C. J.: Statistical characterization of the growth and spatial scales of the substorm onset arc, J. Geophys. Res.-Space, 120, 8503-8516, https://doi.org/10.1002/2015JA021470, 2015.

Kalmoni, N. M. E., Rae, I. J., Murphy, K. R., Forsyth, C., Watt, C. E. J., and Owen, C. J.: Statistical azimuthal structuring of the substorm onset arc: Implications for the onset mechanism, Geophys. Res. Lett., 44, 2078-2087, https://doi.org/10.1002/2016GL071826, 2017.

Kauristie, K., Pulkkinen, T. I., Huuskonen, A., Pellinen, R. J., Opgenoorth, H. J., Baker, D. N., Korth, A., and Syrjäsuo, M.: Auroral precipitation fading before and at substorm onset: ionospheric and geostationary signatures, Ann. Geophys., 15, 967983, https://doi.org/10.1007/s00585-997-0967-4, 1997.

Kauristie, K., Sergeev, V. A., Kubyshkina, M., Pulkkinen, T. I., Angelopoulos, V., Phan, T., Lin, R. P., and Slavin, J. A.: Ionospheric current signatures of transient plasma sheet flows, J. Geophys. Res., 105, 10677-10690, https://doi.org/10.1029/1999JA900487, 2000.

Kazama, Y. and Mukai, T.: Multiple energy-dispersed ion signatures in the near-Earth magnetotail: Geotail observation, Geophys. Res. Lett., 30, 1384, https://doi.org/10.1029/2002GL016637, 2003.

Kepko, L.: Evaluating the role of pre-onset streamers in substorm onset and development, 12th International Conference on Substorms, Ise-Shima, Japan, 10-14 November 2014.

Kepko, L., Spanswick, E., Angelopoulos, V., Donovan, E., McFadden, J., Glassmeier, K.-H., Raeder, J., and Singer, H. J.: Equatorward moving auroral signatures of a flow burst observed prior to auroral onset, Geophys. Res. Lett., 36, L24104, https://doi.org/10.1029/2009GL041476, 2009.

Lessard, M. R., Lotko, W., LaBelle, J., Peria, W., Carlson, C. W., Creutzberg, F., and Wallis, D. D.: Ground and satellite observations of the evolution of growth phase auroral arcs, J. Geophys. Res., 112, A09304, https://doi.org/10.1029/2006JA011794, 2007.

Liang, J., Donovan, E. F., Liu, W. W., Jackel, B., Syrjäsuo, M., Mende, S. B., Frey, H. U., Angelopoulos, V., and Connors, M.: Intensification of preexisting auroral arc at substorm expansion phase onset: Wave-like disruption during the first tens of seconds, Geophys. Res. Lett., 35, L17S19, https://doi.org/10.1029/2008GL033666, 2008.

Liu, J., Angelopoulos, V., Kubyshkina, M., McFadden, J., Glassmeier, K.-H., and Russell, C. T.: Revised timing and onset location of two isolated substorms observed by Time History of Events and Macroscale Interactions During Substorms (THEMIS), J. Geophys. Res., 116, A00I17, https://doi.org/10.1029/2010JA015877, 2011.

Liu, W. W., Liang, J., and Donovan, E. F.: Interaction between kinetic ballooning perturbation and thin current sheet: Quasi-electrostatic field, local onset, and global characteristics, Geophys. Res. Lett., 35, L20107, https://doi.org/10.1029/2008GL035757, 2008.

Lui, A. T. Y.: Current disruption in the Earth's magnetosphere: Observations and models, J. Geophys. Res., 101, 13067-13088, https://doi.org/10.1029/96JA00079, 1996.

Lui, A. T. Y.: Revisiting Time History of Events and Macroscale Interactions during Substorms (THEMIS) substorm events implying magnetic reconnection as the substorm trigger, J. Geophys. Res., 116, A03211, https://doi.org/10.1029/2010JA016078, 2011.

Lyons, L. R., Voronkov, I. O., Donovan, E. F., and Zesta, E.: Relation of substorm breakup arc to other growth-phase auroral arcs, J. Geophys. Res., 107, 1390, https://doi.org/10.1029/2002JA009317, 2002.

Lyons, L. R., Nishimura, Y., Shi, Y., Zou, S., Kim, H.-J., Angelopoulos, V., Heinselman, C., Nicolls, M. J., and Fornacon, K.-H.: Substorm triggering by new plasma intrusion: Incoherentscatter radar observations, J. Geophys. Res., 115, A07223, https://doi.org/10.1029/2009JA015168, 2010.

Machida, S., Miyashita, Y., Ieda, A., Nishida, A., Mukai, T., Saito, Y., and Kokubun, S.: GEOTAIL observations of flow velocity and north-south magnetic field variations in the near and middistant tail associated with substorm onsets, Geophys. Res. Lett., 26, 635-638, https://doi.org/10.1029/1999GL900030, 1999.

Machida, S., Ieda, A., Mukai, T., Saito, Y., and Nishida, A.: Statistical visualization of the Earth's magnetotail during substorms by means of multidimensional superposed epoch analysis with Geotail data, J. Geophys. Res., 105, 25291-25303, https://doi.org/10.1029/2000JA900064, 2000.

Machida, S., Miyashita, Y., Ieda, A., Nosé, M., Nagata, D., Liou, K., Obara, T., Nishida, A., Saito, Y., and Mukai, T.: Statistical visualization of the Earth's magnetotail based on Geotail data and the implied substorm model, Ann. Geophys., 27, 1035-1046, https://doi.org/10.5194/angeo-27-1035-2009, 2009.

Machida, S., Miyashita, Y., Ieda, A., Nosé, M., Angelopoulos, V., and McFadden, J. P.: Statistical visualization of the Earth's magnetotail and the implied mechanism of substorm triggering based on superposed-epoch analysis of THEMIS data, Ann. Geophys., 32, 99-111, https://doi.org/10.5194/angeo-32-99-2014, 2014.

Markwardt, C. B.: Non-linear least squares fitting in IDL with MPFIT, in: Proceedings of Astronomical Data Analysis Software and Systems XVIII, Astronomical Society of the Pacific Conference Series, 411, edited by: Bohlender, D., Durand, D., and Dowler, P., Astronomical Society of the Pacific, San Francisco, USA, 251-254, 2009.

McFadden, J. P., Carlson, C. W., Larson, D., Ludlam, M., Abiad, R., Elliott, B., Turin, P., Marckwordt, M., and Angelopoulos, V.: The THEMIS ESA plasma instrument and in-flight calibration, 
Space Sci. Rev., 141, 277-302, https://doi.org/10.1007/s11214008-9440-2, 2008.

Mende, S. B., Harris, S. E., Frey, H. U., Angelopoulos, V., Russell, C. T., Donovan, E., Jackel, B., Greffen, M., and Peticolas, L. M.: The THEMIS array of ground-based observatories for the study of auroral substorms, Space Sci. Rev., 141, 357-387, https://doi.org/10.1007/s11214-008-9380-x, 2008.

Mende, S., Angelopoulos, V., Frey, H. U., Donovan, E., Jackel, B., Glassmeier, K.-H., McFadden, J. P., Larson, D., and Carlson, C. W.: Timing and location of substorm onsets from THEMIS satellite and ground based observations, Ann. Geophys., 27, 28132830, https://doi.org/10.5194/angeo-27-2813-2009, 2009.

Mende, S. B., Frey, H. U., Angelopoulos, V., and Nishimura, Y.: Substorm triggering by poleward boundary intensification and related equatorward propagation, J. Geophys. Res., 116, A00I31, https://doi.org/10.1029/2010JA015733, 2011.

Miyashita, Y., Machida, S., Mukai, T., Saito, Y., Tsuruda, K., Hayakawa, H., and Sutcliffe, P. R.: A statistical study of variations in the near and middistant magnetotail associated with substorm onsets: GEOTAIL observations, J. Geophys. Res., 105, 15913-15930, https://doi.org/10.1029/1999JA000392, 2000.

Miyashita, Y., Machida, S., Kamide, Y., Nagata, D., Liou, K., Fujimoto, M., Ieda, A., Saito, M. H., Russell, C. T., Christon, S. P., Nosé, M., Frey, H. U., Shinohara, I., Mukai, T., Saito, Y., and Hayakawa, H.: A state-of-the-art picture of substormassociated evolution of the near-Earth magnetotail obtained from superposed epoch analysis, J. Geophys. Res., 114, A01211, https://doi.org/10.1029/2008JA013225, 2009.

Miyashita, Y., Hiraki, Y., Angelopoulos, V., Ieda, A., and Machida, S.: Development of the near-Earth magnetotail and the auroral arc associated with substorm onset: Evidence for a new model, American Geophysical Union Fall Meeting, San Francisco, USA, 14-18 December 2015, SM51E-2597, 2015.

Moré, J. J.: The Levenberg-Marquardt algorithm: Implementation and theory, in: Numerical Analysis, Lecture Notes in Mathematics, 630, edited by: Watson, G. A., Springer, Berlin, Heidelberg, Germany, 105-116, https://doi.org/10.1007/BFb0067700, 1978.

Motoba, T. and Hirahara, M.: High-resolution auroral acceleration signatures within a highly dynamic onset arc, Geophys. Res. Lett., 43, 1793-1801, https://doi.org/10.1002/2015GL067580, 2016.

Motoba, T., Hosokawa, K., Kadokura, A., and Sato, N.: Magnetic conjugacy of northern and southern auroral beads, Geophys. Res. Lett., 39, L08108, https://doi.org/10.1029/2012GL051599, 2012.

Motoba, T., Ohtani, S., Anderson, B. J., Korth, H., Mitchell, D., Lanzerotti, L. J., Shiokawa, K., Connors, M., Kletzing, C. A., and Reeves, G. D.: On the formation and origin of substorm growth phase/onset auroral arcs inferred from conjugate spaceground observations, J. Geophys. Res.-Space, 120, 8707-8722, https://doi.org/10.1002/2015JA021676, 2015.

Murphy, K. R., Mann, I. R., Rae, I. J., Waters, C. L., Anderson, B. J., Milling, D. K., Singer, H. J., and Korth, H.: Reduction in field-aligned currents preceding and local to auroral substorm onset, Geophys. Res. Lett., 39, L15106, https://doi.org/10.1029/2012GL052798, 2012.

Murphy, K. R., Mann, I. R., Rae, I. J., Waters, C. L., Frey, H. U., Kale, A., Singer, H. J., Anderson, B. J., and Korth, H.: The detailed spatial structure of field-aligned currents comprising the substorm current wedge, J. Geophys. Res.-Space, 118, 77147727, https://doi.org/10.1002/2013JA018979, 2013.

Murphy, K. R., Miles, D. M., Watt, C. E. J., Rae, I. J., Mann, I. R., and Frey, H. U.: Automated determination of auroral breakup during the substorm expansion phase using allsky imager data, J. Geophys. Res.-Space, 119, 1414-1427, https://doi.org/10.1002/2013JA018773, 2014a.

Murphy, K. R., Mann, I. R., Rae, I. J., Walsh, A. P., and Frey, H. U.: Inner magnetospheric onset preceding reconnection and tail dynamics during substorms: Can substorms initiate in two different regions?, J. Geophys. Res.-Space, 119, 9684-9701, https://doi.org/10.1002/2014JA019795, 2014b.

Nagai, T., Fujimoto, M., Saito, Y., Machida, S., Terasawa, T., Nakamura, R., Yamamoto, T., Mukai, T., Nishida, A., and Kokubun, S.: Structure and dynamics of magnetic reconnection for substorm onsets with Geotail observations, J. Geophys. Res., 103, 4419-4440, https://doi.org/10.1029/97JA02190, 1998.

Nagata, D., Machida, S., Ohtani, S., Mende, S. B., Saito, Y., and Mukai, T.: Remote sensing of a near-Earth neutral line during the 5 October 2000 substorm, Ann. Geophys., 24, 3497-3505, https://doi.org/10.5194/angeo-24-3497-2006, 2006.

Nakamura, R., Baumjohann, W., Schödel, R., Brittnacher, M., Sergeev, V. A., Kubyshkina, M., Mukai, T., and Liou, K.: Earthward flow bursts, auroral streamers, and small expansions, J. Geophys. Res., 106, 10791-10802, https://doi.org/10.1029/2000JA000306, 2001.

Nishimura, Y., Lyons, L., Zou, S., Angelopoulos, V., and Mende, S.: Substorm triggering by new plasma intrusion: THEMIS all-sky imager observations, J. Geophys. Res., 115, A07222, https://doi.org/10.1029/2009JA015166, 2010.

Nishimura, Y., Lyons, L. R., Angelopoulos, V., Kikuchi, T., Zou, S., and Mende, S. B.: Relations between multiple auroral streamers, pre-onset thin arc formation, and substorm auroral onset, J. Geophys. Res., 116, A09214, https://doi.org/10.1029/2011JA016768, 2011.

Nishimura, Y., Lyons, L. R., Shiokawa, K., Angelopoulos, V., Donovan, E. F., and Mende, S. B.: Substorm onset and expansion phase intensification precursors seen in polar cap patches and arcs, J. Geophys. Res.-Space, 118, 2034-2042, https://doi.org/10.1002/jgra.50279, 2013a.

Nishimura, Y., Lyons, L. R., Xing, X., Angelopoulos, V., Donovan, E. F., Mende, S. B., Bonnell, J. W., and Auster, U.: Identifying the magnetotail source region leading to preonset poleward boundary intensifications, J. Geophys. Res.-Space, 118, 43354340, https://doi.org/10.1002/jgra.50407, 2013 b.

Nishimura, Y., Lyons, L. R., Xing, X., Angelopoulos, V., Donovan, E. F., Mende, S. B., Bonnell, J. W., and Auster, U.: Tail reconnection region versus auroral activity inferred from conjugate ARTEMIS plasma sheet flow and auroral observations, J. Geophys. Res.-Space, 118, 5758-5766, https://doi.org/10.1002/jgra.50549, 2013c.

Nishimura, Y., Lyons, L. R., Nicolls, M. J., Hampton, D. L., Michell, R. G., Samara, M., Bristow, W. A., Donovan, E. F., Spanswick, E., Angelopoulos, V., and Mende, S. B.: Coordinated ionospheric observations indicating coupling between preonset flow bursts and waves that lead to substorm onset, J. Geophys. Res.-Space, 119, 3333-3344, https://doi.org/10.1002/2014JA019773, 2014. 
Nishimura, Y., Yang, J., Pritchett, P. L., Coroniti, F. V., Donovan, E. F., Lyons, L. R., Wolf, R. A., Angelopoulos, V., and Mende, S. V.: Statistical properties of substorm auroral onset beads/rays, J. Geophys. Res.-Space, 121, 8661-8676, https://doi.org/10.1002/2016JA022801, 2016.

Pellinen, R. J. and Heikkila, W. J.: Observations of auroral fading before breakup, J. Geophys. Res., 83, 4207-4217, https://doi.org/10.1029/JA083iA09p04207, 1978.

Rae, I. J., Mann, I. R., Angelopoulos, V., Murphy, K. R., Milling, D. K., Kale, A., Frey, H. U., Rostoker, G., Russell, C. T., Watt, C. E. J., Engebretson, M. J., Moldwin, M. B., Mende, S. B., Singer, H. J., and Donovan, E. F.: Near-Earth initiation of a terrestrial substorm, J. Geophys. Res., 114, A07220, https://doi.org/10.1029/2008JA013771, 2009.

Rae, I. J., Watt, C. E. J., Mann, I. R., Murphy, K. R., Samson, J. C., Kabin, K., and Angelopoulos, V.: Optical characterization of the growth and spatial structure of a substorm onset arc, J. Geophys. Res., 115, A10222, https://doi.org/10.1029/2010JA015376, 2010.

Rae, I. J., Watt, C. E. J., Murphy, K. R., Frey, H. U., Ozeke, L. G., Milling, D. K., and Mann, I. R.: The correlation of ULF waves and auroral intensity before, during and after substorm expansion phase onset, J. Geophys. Res., 117, A08213, https://doi.org/10.1029/2012JA017534, 2012.

Rae, I. J., Murphy, K. R., Watt, C. E. J., Mann, I. R., Yao, Z., Kalmoni, N. M. E., Forsyth, C., and Milling, D. K.: Using ultra-low frequency waves and their characteristics to diagnose key physics of substorm onset, Geosci. Lett., 4, 23, https://doi.org/10.1186/s40562-017-0089-0, 2017.

Saito, M. H., Miyashita, Y., Fujimoto, M., Shinohara, I., Saito, Y., Liou, K., and Mukai, T.: Ballooning mode waves prior to substorm-associated dipolarizations: Geotail observations, Geophys. Res. Lett., 35, L07103, https://doi.org/10.1029/2008GL033269, 2008.
Sakaguchi, K., Shiokawa, K., Ieda, A., Nomura, R., Nakajima, A., Greffen, M., Donovan, E., Mann, I. R., Kim, H., and Lessard, M.: Fine structures and dynamics in auroral initial brightening at substorm onsets, Ann. Geophys., 27, 623-630, https://doi.org/10.5194/angeo-27-623-2009, 2009a.

Sakaguchi, K., Shiokawa, K., and Donovan, E.: Azimuthal structures of ray auroras at the beginning of auroral substorms, Geophys. Res. Lett., 36, L23106, https://doi.org/10.1029/2009GL041252, 2009b.

The Space Sciences Laboratory: University of California, Berkeley, available at: http://themis.ssl.berkeley.edu/, last access: 17 October 2018 .

Tomé, A. R. and Miranda, P. M. A.: Piecewise linear fitting and trend changing points of climate parameters, Geophys. Res. Lett., 31, L02207, https://doi.org/10.1029/2003GL019100, 2004.

Tomé, A. R. and Miranda, P. M. A.: Continuous partial trends and low-frequency oscillations of time series, Nonlin. Processes Geophys., 12, 451-460, https://doi.org/10.5194/npg-12-451-2005, 2005.

Tsyganenko, N. A.: Modeling the Earth's magnetospheric magnetic field confined within a realistic magnetopause, J. Geophys. Res., 100, 5599-5612, https://doi.org/10.1029/94JA03193, 1995.

Virginia Polytechnic Institute and State University: available at: http://vt.superdarn.org/, last access: 17 October 2018.

World Data Center for Geomagnetis: Kyoto, available at: http://wdc. kugi.kyoto-u.ac.jp/, last access: 17 October 2018.

Xing, X., Lyons, L., Nishimura, Y., Angelopoulos, V., Larson, D., Carlson, C., Bonnell, J., and Auster, U.: Substorm onset by new plasma intrusion: THEMIS spacecraft observations, J. Geophys. Res., 115, A10246, https://doi.org/10.1029/2010JA015528, 2010. 